\title{
Rippling and crumpling in disordered free-standing graphene
}

\author{
I. V. Gornyi ${ }^{1,2,3,5}$, V. Yu. Kachorovskii ${ }^{1,2,3,5}$, and A. D. Mirlin ${ }^{1,3,4,5}$ \\ ${ }^{1}$ Institut für Nanotechnologie, Karlsruhe Institute of Technology, 76021 Karlsruhe, Germany \\ 2 A.F.Ioffe Physico-Technical Institute, 194021 St. Petersburg, Russia \\ ${ }^{3}$ Institut für Theorie der kondensierten Materie, Karlsruhe Institute of Technology, 76128 Karlsruhe, Germany \\ 4 Petersburg Nuclear Physics Institute, 188300, St.Petersburg, Russia \\ 5 L.D. Landau Institute for Theoretical Physics, Kosygina street 2, 119334 Moscow, Russia
}

(Dated: October 12, 2018)

\begin{abstract}
Graphene is a famous realization of elastic crystalline two-dimensional (2D) membrane. Thermal fluctuations of a 2D membrane tend to destroy the long-range order in the system. Such fluctuations are stabilized by strong anharmonicity effects, which preserve thermodynamic stability. The anharmonic effects demonstrate critical behaviour on scales larger than the Ginzburg scale. In particular, clean suspended flake of graphene shows a power-law increase of the bending rigidity with the system size, $\varkappa \propto L^{\eta}$, due to anharmonic interaction between in-plane and out-of-plane (flexural) phonon modes. We demonstrate that random fluctuations of membrane curvature caused by static disorder may change dramatically the scaling of the bending rigidity and lead to a non-monotonous dependence of $\varkappa$ on $L$. We derive coupled renormalization-group equations describing combined flow of $\varkappa$ and effective disorder strength $b$, find a critical curve $b(\varkappa)$ separating flat and crumpled phases, and explore the behavior of disorder in the flat phase. Deep in the flat phase, disorder decays in a power-law way at scales larger than the Ginzburg length which therefore sets a characteristic size for the ripples - static out-of-plane deformations observed experimentally in suspended graphene. We find that in the limit $L \rightarrow \infty$ ripples are characterized by anomalous exponent $2 \eta$ in contrast to dynamical fluctuations governed by $\eta$. For sufficiently strong disorder, there exists an intermediate range of spatial scales where ripples decay much slower, with exponent $\eta / 4$. In the near-critical regime, disorder first increase with $L$, then reaches a maximum and starts to decrease. In this case, the membrane shows fractal properties implying a multiple folding starting from a certain length scale $L_{1}$ and finally flattens at a much larger scale $L_{2}$ (which diverges at criticality). We conclude the paper by a comparison of our results with available experimental data on graphene ripples.
\end{abstract}

PACS numbers: 72.80.Vp, 73.23.Ad, 73.63.Bd

\section{INTRODUCTION}

Graphene, a single monolayer of graphite,,$\underline{\underline{1}-\underline{3}}$ has attracted enormous interest in the last decade (for review, see Refs. 4-10). From the fundamental point of view, this interest is largely motivated by quasirelativistic character of its spectrum: charge carriers in graphene are twodimensional (2D) massless Dirac fermions. This leads to a variety of remarkable phenomena. In particular, graphene is a unique example of a system where essentially quantum phenomena such as the quantum Hall effect can be observed up to the room temperature. 11 In view of applications, the technological breakthrough in fabrication of flat monolayer 2D crystals offers new opportunities in the future nanoelectronics. Remarkably, suspended graphene demonstrates extremely high roomtemperature mobility $\underline{\underline{12}-23}$ as high as $1.2 \cdot 10^{5} \mathrm{~cm}^{2} / \mathrm{Vs}$ and therefore is considered as a most perspective candidate for the carbon-based nanoelectronics.

Elastic properties of graphene are also quite amazing. Free-standing graphene is an outstanding example of an elastic crystalline two-dimensional (2D) membrane with a high bending rigidity $\varkappa \simeq 1 \mathrm{eV}$. The most important feature distinguishing such a membrane from conventional $2 \mathrm{D}$ semiconductor systems is the existence of specific type of the out-of-plane phonon modes, so called flexural phonons $\stackrel{24}{2}$ In the harmonic approximation the energy of out-of-plane deformation reads

$$
E=\frac{1}{2} \int d \mathbf{x}\left[\rho \dot{h}^{2}+\varkappa(\Delta h)^{2}\right]
$$

where $h(\mathbf{x})$ is out-of-plane distortion and $\rho$ is the mass density per unit square. From Eq. (10) we find frequency of the flexural phonons

$$
\omega_{\mathbf{q}}=D q^{2},
$$

where $D=\sqrt{\varkappa / \rho}$. Hence, in contrast to in-plane acoustic phonons, whose frequency scales as $q$, the flexural mode is very soft and, consequently, the out-of-plane thermal fluctuations of $h(\mathbf{r})$ are very large. As a consequence, flexural phonons serve as a very effective scattering mechanism for electrons (for discussion of different aspects of electron-phonon scattering in graphene see Refs. 25 45).

A remarkable property of flexural phonons is a crucial role played by anharmonic effects. In particular, golden-rule calculation of scattering rate on the deformation potential created by flexural phonons leads, in the harmonic approximation and with electrostatic screening taken into account, to values of the electrical conductivity that are two to three orders of magnitude lower than experimentally observed. This drastic discrepancy implies the existence of a certain mechanism that strongly suppresses out-of-plane modes. As was demonstrated in Ref. 42, taking into account anharmonic interaction 
between flexural and in-plane phonons dramatically reduces the electron-phonon scattering, yielding values of the electrical conductivity that are in good agreement with experimental findings of Ref. 14. Hence, a comparison of theoretical results with transport measurements demonstrates that anharmonic effects are extremely important in suspended graphene.

Suppression of scattering because of anharmonicity is very favourable for fabrication of ultra-high-mobility graphene structures. Further, the anharmonicity governs lattice thermal transport in suspended graphene, which is currently a subject of intense experimental $46-48$ and theoretical research (see, e.g. Ref. 49 and references therein). Moreover, anharmonicity plays a key role for the fundamental issue of the thermodynamic stability of graphene membrane. Indeed, due to the softness of flexural-phonon modes they might be expected to be very efficient in inducing strong thermal out-of-plane fluctuations and thus destroying the membrane 50,51 by driving it into the so-called crumpled phase ${ }^{24}$ This question was intensively discussed in the literature more than two decades ago $24,52-68$ in connection with biological membranes, polymerized layers and some inorganic surfaces. The interest to this topic has been renewed more recently $\underline{69}-76$ after discovery of graphene.

It was found ${ }^{52}-58$ that anharmonic coupling of in-plane and out-of-plane phonons stabilizes the membrane for sufficiently low temperatures $T$, so that the membrane is in the flat phase at relatively low $T$ and undergoes the crumpling transition with increasing $T$. The main dimensionless parameter characterizing the state of the $2 \mathrm{D}$ membrane is the ratio of the bending rigidity $\varkappa$ to the temperature. For graphene this ratio for room temperature is quite large, $\varkappa / T \simeq 30$. This reflects a remarkable rigidity of graphene and implies that graphene remains in the flat phase up to the temperatures several times higher than the room temperature. Moreover, interaction between flexural and in-plane phonons leads to a power-law renormalization of the bending rigidity $57,59,65$

$$
\varkappa \rightarrow \varkappa(q) \propto q^{-\eta}, \text { for } q \rightarrow 0
$$

with a certain critical index $\eta$. Physically, the increase of the bending rigidity, Eq. (3), is a manifestation of the tendency of the membrane towards the flat phase.

Development of a controllable analytical treatment of renormalization of the bending rigidity and of the crumpling transition is not an easy task. The central problem is the absence of a small parameter that would control the analysis in the physically relevant case of a membrane with dimension $D=2$ embedded into the space with dimension $d=3$. For a membrane with arbitrary $D$ and $d$, a systematic treatment turns out to be possible in two cases: for $4-D=\epsilon \ll 1$ and for $d_{c}=d-D \gg 1$. In both limits, there exists a small parameter that controls calculations: $\epsilon$ in the first case, and $1 / d_{c}$ in the second case. In particular, a theory of crumpling transition for $D=2$ and $d_{c} \gg 1$ was developed in Ref. 55. 58, while a renormalization-group $(\mathrm{RG})$ treatment of the membrane elastic coefficients in the limit $\epsilon \ll 1$ was first discussed in Ref. 54,57. The value of the critical exponent $\eta$ characterising the flat phase was found to be

$$
\eta \simeq \frac{2}{d_{c}}
$$

for $D=2$ and $d_{c} \gg 1,, 55,58$ and

$$
\eta \simeq \frac{12 \epsilon}{24+d_{c}}
$$

for $\epsilon \ll 1.57$

The scaling of the bending rigidity exactly at the crumpling transition point is characterised by another critical exponent $\eta_{\mathrm{cr}}$. This exponent determines the fractal (Hausdorff) dimension of the membrane at criticality, $D_{H}=2 D /\left(4-D-\eta_{\mathrm{cr}}\right)$. It turns out that for $D=2$ and $d_{c} \gg 1$ the exponent $\eta_{\mathrm{cr}}$ coincides with $\eta$, Eq. (4), in the leading order in $1 / d_{c}$. On the other hand, in the vicinity of $D=4$ the exponent $\eta_{\mathrm{cr}}$ was found to be essentially different from $\eta$, Eq. (5), and scaling as $\epsilon^{3}$, see Refs. 59,60.

Further approximate calculation schemes (that become controllable for $d_{c} \gg 1$ ) have been developed, such as the self-consistent screening approximation (SCSA) 65 and the "non-perturbative renormalization group" 69. After extrapolation to the physical dimensionality, the corresponding results yield $\eta=0.821$ and $\eta=0.849$, respectively. Clearly, the extrapolation is not controlled parametrically; the scattering between the above values may serve as a rough estimate of their accuracy. Numerical simulations of the problem gave values $\eta=0.60 \pm 0.10$ and $\eta=0.72 \pm 0.04$ (see Ref. 63 and Ref. 68, respectively).

In the present paper we explore the interplay of a static disorder and anharmonicities in a graphene membrane. Some aspects of such a problem have been discussed in the literature in a general context of the membrane physics $61,62,64,66,67$. In particular, it was concluded in Refs. 61, 62, 66 that for $D=4-\epsilon$ with small $\epsilon$ the flat phase is stable with respect to various types of disorder. On the other hand, it was argued in Ref. 64 that, in the leading order in $1 / d_{c}$, a flat phase of a $2 \mathrm{D}$ membrane is destroyed already by an infinitely small impurity-induced disorder (in-plane quenched random deformations). The later result, if applicable in the physical case $d=3$, would imply an instability of the graphene membrane with respect to an arbitrarily weak disorder. The authors of Ref. 64 speculated that high-order terms with respect to $1 / d_{c}$ might cure such an instability. In Ref. 67, influence of randomness on the flat phase was treated within the SCSA, with a focus on a long-range disorder.

Although effects of disorder in the flat phase (both for $D=4-\epsilon$ and $D=2$ ) were discussed in a number of publications, the problem of crumpling transition in a disordered membrane still remains a challenge. This problem has a close relation to formation of ripples which are the static out-of-plane random deformations of membrane. In other words, ripples look like 
frozen flexural phonons. In graphene, such deformations with the height $3-10 \AA$ and typical spatial scale about several hundred angstroms were observed in a number of experiments, $\underline{17,23,77-85}$ While several recent theoretical papers discussed graphene ripples, $\underline{30,40,71,72,85}$ development of a systematic theory of their formation (which would explain the mechanism of rippling and predict key parameters of the emerging structure) remains a challenging problem.

In the present work, we study the crumpling transition and the rippling in graphene with a static quenched disorder. We perform a detailed RG analysis for the out-ofplane ("random curvature") disorder model. Previously, the RG treatment of disorder in membranes $\underline{61,62,66}$ has been performed only for dimensionality $D=4-\epsilon$, which cannot be directly employed to the physical graphene samples. We assume that the membrane dimensionality $D$ is equal (or close) to 2 and use the $1 / d_{c^{-}}$expansion which allows us to control the theory and to derive coupled RG equations describing a combined flow of $\varkappa$ and effective strength $b$ of the out-of-plane disorder. In this way, we establish the phase diagram by determining a critical curve $b(\varkappa)$ separating the flat and crumpled phases. We demonstrate that, even deep in the flat phase, random fluctuation of membrane tension caused by the static disorder may strongly change the critical behavior of the bending rigidity. We discuss in detail the behavior of disorder in the flat phase.

Far from the critical curve, i.e. deep in the flat phase, disorder decays in a power-law way at the scales larger than the Ginzburg length which therefore sets a characteristic size for the ripples - static frozen outof-plane deformations observed experimentally in suspended graphene. We find that in the limit $L \rightarrow \infty$ static and dynamic correlation functions of $\nabla h$ behave as $L^{-2 \eta}$ and $L^{-\eta}$, respectively. Hence, ripples are characterized by the anomalous exponent $2 \eta$ in contrast to dynamical fluctuations governed by $\eta$. Furthermore, we show that, for sufficiently strong disorder, there exists an intermediate range of spatial scales where ripples decay much slower, with exponent $\eta / 4$. One of remarkable results is that the RG flow of coupling constants may be essentially non-monotonous. Specifically, for sufficiently strong disorder (close to the critical curve), the bending rigidity decreases at the first stage of the renormalization, reaches its minimum, and then starts to grow. In this near-critical regime the disorder also changes in a non-monotonous fashion: it first increases very slowly (logarithmically) with the spatial scale $L$, then reaches a maximum at a certain scale $L_{r}$ and, finally, decreases according to a power law at larger scales. In this case, the membrane shows fractal properties which imply its multiple folding starting form a certain length scale $L_{1}$ and flattens at a much larger scale $L_{2}$.

We also briefly discuss an in-plane disorder and show that it is irrelevant in the RG sense, unless its correlation function is highly singular at small momenta, i.e., unless it is too long-ranged. Thus, the above conclusions remain valid also in the presence of in-plane disorder. The inplane disorder may, however, affect essentially the bare value of the out-of-plane disorder at the atomic scales, which serves as a starting point for the RG analysis.

We conclude the paper by a comparison of our results with available experimental data on graphene ripples. While the main focus of the paper is on graphene, the developed theory is quite general and is expected to be applicable to other crystalline membranes as well. These include biological membranes like those of red blood cells, $\underline{86}$ oxidised graphite or graphene, $\stackrel{87}{=}$ graphane,,$\stackrel{88}{\underline{1}}$ molybdenum disulphide,, 89 and boron nitride 90 membranes. Further examples may likely emerge in near future, in view of current active works on engineering of $2 \mathrm{D}$ materials and structures.

\section{FORMULATION OF THE PROBLEM AND MEAN-FIELD ANALYSIS}

To begin with, we note that the energy of membrane consists of kinetic and elastic contributions. In this paper, we treat the problem semiclassically (see Appendix $\mathrm{A}$ for discussion of the region of applicability of the quasiclassical approximation). The kinetic energy depends on momenta only. Within the semiclassical approximation, the phonon momenta can thus be integrated out from the very beginning. In what follows we neglect the kinetic term (see Refs. 73,74,76 for a discussion of some effects related to this term) and focus on the study of the elastic terms.

We start from the clean case and consider a general Landau-Ginzburg form of the elastic free energy ${ }^{\underline{54}}$

$$
\begin{aligned}
& F=\int d^{D} x\left\{\frac{w}{2}\left(\partial_{\alpha} \partial_{\alpha} \mathbf{R}\right)^{2}-\frac{t}{2}\left(\partial_{\alpha} \mathbf{R} \partial_{\alpha} \mathbf{R}\right)\right. \\
& \left.+u\left(\partial_{\alpha} \mathbf{R} \partial_{\beta} \mathbf{R}\right)^{2}+v\left(\partial_{\alpha} \mathbf{R} \partial_{\alpha} \mathbf{R}\right)^{2}\right\}
\end{aligned}
$$

Here $D$ is the dimension of the membrane, $d$ is the dimension of the embedding space and $\alpha, \beta=1, \ldots, D$. In principle, Eq. (6) allows one to describe both flat and crumpled phases of the membrane. In the latter case, one should include an additional term that prevents selfintersections. In this paper, we focus on the flat phase, so that we omit this term. The $d$-dimensional vector $\mathbf{R}$ depends on the point of the $D$-dimensional reference space, $\mathbf{x}=\left(x_{1}, \ldots, x_{D}\right)$, i.e., $\mathbf{R}=\mathbf{R}(\mathbf{x})$ (see Fig. 1). Equation (6) can be obtained by following considerations. First, the translational invariance implies that the free energy depends on $\mathbf{R}$ via the derivatives $\partial_{\alpha} \mathbf{R}$ only. Second, the rotational invariance requires that the energy should be scalar with respect to both the embedding $(\mathbf{R})$ space and the reference $(\mathbf{x})$ space. Finally, keeping the leading terms in the expansion in the field $\partial_{\alpha} \mathbf{R}$ and in gradients yields Eq. (6).

Following Ref. 54, we first consider the mean field approximation which amounts to the linear ansatz,

$$
\mathbf{R}=\xi_{0} \mathbf{x}
$$




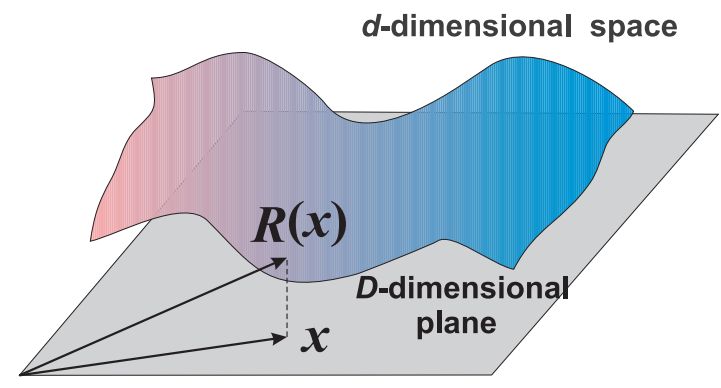

FIG. 1: Membrane with dimension $D$ is embedded into a space with dimension $d$. A point on the membrane surface is labeled by a $d$-dimensional vector $\mathbf{R}$ which depends on vector x belonging to an arbitrary $D$-dimensional reference plane.

This yields the free energy of Landau type,

$$
F \propto-\xi_{0}^{2} t+2 \xi_{0}^{4}(u+D v) .
$$

Next, we find $\xi_{0}$ by minimization of the free energy (77):

$$
\partial F / \partial \xi_{0}=0 \Rightarrow \xi_{0}^{2}=\left\{\begin{array}{cl}
\frac{t}{4(u+D v)}, & \text { for } t>0 \\
0, & \text { for } t<0
\end{array}\right.
$$

The case with $\xi_{0}^{2}>0$ corresponds to the flat phase, while for $\xi_{0}^{2}=0$ the manifold $\mathbf{R}(\mathbf{x})$ shrinks to a point $\mathbf{R}=0$, which implies that the membrane is crumpled. (Taking self-avoidance into account would lead to a finite radius of the crumpled membrane ${ }^{54}$ ) Assuming (in spirit of Landau theory of phase transitions) that $t \propto T_{c}-T$, we find that $\xi_{0}^{2} \propto T_{c}-T$. Hence, the model Eq. (6) shows a crumpling transition at $T=T_{c}$ already within the mean-field approximation.

We assume now that the system is in the flat phase $T<$ $T_{c}$ and take into account thermal fluctuations around the mean-field solution. For this purpose, we write

$$
\mathbf{R}=\xi_{0} \mathbf{r},
$$

where

$$
\mathbf{r}=\mathbf{x}+\mathbf{u}+\mathbf{h}
$$

and vectors

$$
\mathbf{u}=\left(u_{1}, \ldots, u_{D}\right), \mathbf{h}=\left(h_{1}, \ldots, h_{d-D}\right)
$$

represents in-plane and out-of-plane displacements, respectively. Substituting Eq. (9) into Eq. (6) and choosing for $\xi_{0}$ the mean-field solution Eq. (8), we find

$$
\begin{aligned}
& F=\int d^{D} x\left\{\frac{\varkappa}{2}(\Delta \mathbf{r})^{2}\right. \\
& \left.+\frac{\mu}{4}\left[\partial_{\alpha} \mathbf{r} \partial_{\beta} \mathbf{r}-\delta_{\alpha \beta}\right]^{2}+\frac{\lambda}{8}\left[\partial_{\gamma} \mathbf{r} \partial_{\gamma} \mathbf{r}-D\right]^{2}\right\} .
\end{aligned}
$$

Here

$$
\varkappa=w \xi_{0}^{2}
$$

is the bending rigidity and

$$
\mu=4 u \xi_{0}^{4}, \lambda=8 v \xi_{0}^{4}
$$

are in-plane elastic constants. We see that these constants as well as $\varkappa$ turn to zero at the transition point: $\mu, \lambda \propto\left(T_{c}-T\right)^{2}, \varkappa \propto T_{c}-T$.

Next, we use the parametrisation (10) and rewrite Eq. (11) in terms of $\mathbf{u}$ and $\mathbf{h}$. We assume that spatial derivatives of fields are small, $\left|\partial_{\alpha} \mathbf{h}\right| \ll 1,\left|\partial_{\alpha} \mathbf{u}\right| \ll 1$, so that one can neglect terms $\partial_{\alpha} \mathbf{u} \partial_{\beta} \mathbf{u}$ compared to $\partial_{\alpha} \mathbf{u}$ in the second and third contributions to the energy (11). Further, one may neglect the term $\varkappa(\Delta \mathbf{u})^{2}$ compared to $\mu\left(\partial_{\alpha} \mathbf{u}_{\beta}\right)^{2}$ and $\lambda\left(\partial_{\alpha} \mathbf{u}_{\alpha}\right)^{2}$ provided that the characteristic length scale of the variation of the membrane displacement, $q^{-1}$, is sufficiently large:

$$
q \ll \min \left(\sqrt{\frac{\mu}{\varkappa}}, \sqrt{\frac{\lambda}{\varkappa}}\right) .
$$

Under these assumption, one can rewrite Eq. (11) for membrane energy in the standard textbook form ${ }^{24}$ :

$$
F=\int d^{D} x\left\{\frac{\varkappa}{2}(\Delta \mathbf{h})^{2}+\mu u_{i j}^{2}+\frac{\lambda}{2} u_{i i}^{2}\right\},
$$

where

$$
u_{\alpha \beta}=\frac{1}{2}\left(\partial_{\alpha} \mathbf{r} \partial_{\beta} \mathbf{r}-\delta_{\alpha \beta}\right) \approx \frac{1}{2}\left(\partial_{\alpha} u_{\beta}+\partial_{\beta} u_{\alpha}+\partial_{\alpha} \mathbf{h} \partial_{\beta} \mathbf{h}\right)
$$

is the deformation tensor.

The following comment is in order here. The question about the range of applicability of the simplified model, Eq. (14) is in fact somewhat more subtle than one might conclude from the above discussion. The point is that the elastic constants $\mu, \lambda$, and $\varkappa$ are strongly renormalized due to anharmonicity and critical fluctuations that it induces. This effect becoming prominent for $q \ll q^{*}$, where $q^{*}$ is the inverse Ginzburg scale, see Eq. (41) below. At such scales, the criterion (13) for neglecting the $\varkappa(\Delta \mathbf{u})^{2}$ term should be modified. In particular, for $D=2$, the screening by critical fluctuations reduces $\mu$ and $\lambda$ to the value $\sim T / \Pi_{\mathbf{q}} \sim \varkappa^{2} q^{2} / T$ (see section [IIB). Hence, $q$ drops out from the estimate Eq. (13) and instead we get the condition $\varkappa \gg T$. As will be demonstrated below, the crumpling transition corresponds to $\varkappa \sim d_{c}^{2} T$, where $d_{c}=d-D$. Hence, the simplified model (14) is sufficient for a description of the crumpling transition provided that $d_{c} \gg 1$. On the other hand, it is exactly the latter condition that we will use to develop a controllable RG treatment for the theory (14). Therefore, neglecting the $\varkappa(\Delta \mathbf{u})^{2}$ term and thus restricting ourselves with the free energy (14) is fully consistent with the subsequent RG analysis.

Let us now introduce a quenched disorder into the model (14). We will mainly focus on the out-of-plane (random curvature) disorder in this paper $\underline{61,66}$ Our motivations for analysing the effect of this type of disorder are twofold. First, we will show that the random-curvature 
disorder influences the RG flow (and thus the crumpling transition) in an essential way. This should be contrasted with the in-plane disorder, which is RG-irrelevant (unless its correlation function is highly singular at low momenta), as will be shown below. Second, such disorder induces static out-of-plane deformations and is thus directly related to the effect of rippling.

The free energy including the random-curvature disorder reads $\underline{61}$

$$
F(\mathbf{u}, \mathbf{h})=\int d^{D} x\left\{\frac{\varkappa}{2}(\Delta \mathbf{h}+\boldsymbol{\beta})^{2}+\mu u_{i j}^{2}+\frac{\lambda}{2} u_{i i}^{2}\right\} .
$$

Here $\boldsymbol{\beta}=\boldsymbol{\beta}(\mathrm{x})$ is a random vector with Gaussian distribution

$$
P(\boldsymbol{\beta})=Z_{\boldsymbol{\beta}}^{-1} \exp \left(-\frac{1}{2 b} \int \beta^{2}(\mathbf{x}) d^{D} \mathbf{x}\right),
$$

where $b$ is the disorder strength and $Z_{\boldsymbol{\beta}}$ is a normalization factor. In order to study the membrane properties in a systematic way, one should take into account fluctuations, thus going beyond the mean-field approximation.

\section{BEYOND MEAN FIELD}

In this Section, we develop an RG treatment of a disordered membrane. Previously, the curvature disorder was treated within the RG approach $\frac{66}{6}$ only in dimensionality $D=4-\epsilon$. We will see below that the structure of the RG near $D=2$ is crucially different from the case $D=4-\epsilon$. A lowest-order perturbative treatment of the curvature disorder in physical dimension $D=2$ was briefly discussed in Ref. 61. However, this consideration is only applicable for short distances (smaller than then the Ginzburg length), whereas the most interesting physics (captured by the RG) developes on larger scales.

Our analysis is based on an expansion around the ordered (flat-membrane) phase, and thus bears analogy to a $\sigma$-model treatment of conventional critical phenomena. In full similarity with the $\sigma$-models, $D=2$ is a logarithmic dimension for the present problem (which manifests in $\varkappa / T$ becoming dimensionless in this case), so that our analysis will be appropriate at or near $D=2$. This is highly favourable, since $D=2$ is exactly the physical dimension of a membrane. To keep the theory under control, we will also assume a large dimensionality of the embedding space, $d_{c} \gg 1$.

\section{A. Renormalization of stretching parameter $\xi$}

Above we rescaled coordinates with the scaling factor $\xi_{0}$ which minimize the free energy within the mean-field approximation. Beyond mean field one should take into account critical fluctuations. Such fluctuations change the optimal scaling factor. To consider this effect, we modify Eq. (10) as follows:

$$
\mathbf{r}=\xi \mathbf{x}+\mathbf{u}+\mathbf{h} .
$$

Equation (18) represents a new rescaling of coordinates. The mean-field approximation corresponds to $\xi=1$. Below, we find that $\xi$ flows away from the mean-field value due to the fluctuations. Substituting Eq. (18) into Eq. (11) and using the same approximations that were done in the course of derivation of Eq. (15), we arrive at the following expression for the free energy:

$$
\begin{aligned}
F & =\frac{D L^{D}(\mu+\lambda D / 2)}{4}\left[\left(\xi^{2}-1\right)^{2}\right. \\
& \left.+\frac{2\left(\xi^{2}-1\right)}{D} \int \frac{d^{D} \mathbf{x}}{L^{D}} \partial_{\alpha} \mathbf{h} \partial_{\alpha} \mathbf{h}\right]+F(\tilde{\mathbf{u}}, \mathbf{h}),
\end{aligned}
$$

where $F(\tilde{\mathbf{u}}, \mathbf{h})$ is given by Eq. (16) with $\mathbf{u}$ replaced by $\tilde{\mathbf{u}}=\xi \mathbf{u}$.

Since the product $\partial_{\alpha} \mathbf{h} \partial_{\alpha} \mathbf{h}$ in Eq. (19) is averaged over the whole volume of the membrane, its fluctuations are negligibly small in the limit $L \rightarrow \infty$. Hence, one can replace

$$
\partial_{\alpha} \mathbf{h} \partial_{\alpha} \mathbf{h} \rightarrow\left\langle\partial_{\alpha} \mathbf{h} \partial_{\alpha} \mathbf{h}\right\rangle,
$$

where $\langle\cdots\rangle$ stands for averaging with the statistical factor $\exp [-F(\tilde{\mathbf{u}}, \mathbf{h}) / T]$. Minimizing thus obtained energy with respect to $\xi$, we find that the optimal value of $\xi$ deviates from the mean field value $\xi=1$ due to the fluctuations,

$$
\xi^{2}=1-\frac{1}{D}\left\langle\partial_{\alpha} \mathbf{h} \partial_{\alpha} \mathbf{h}\right\rangle .
$$

In order to calculate $\left\langle\partial_{\alpha} \mathbf{h} \partial_{\alpha} \mathbf{h}\right\rangle$, we notice that $F(\tilde{\mathbf{u}}, \mathbf{h})$ contains linear, quadratic, cubic, and quartic terms with respect to variables $\mathbf{h}$ and $\mathbf{u}$. In this section, we neglect cubic and quartic terms, thus neglecting anharmonicity. The anharmonicity-related effects will be included in the next section in the framework of the RG formalism. In the harmonic approximation, the $\mathbf{u}$-dependent terms in the free energy do not couple with $\mathbf{h}$-dependent ones and, therefore, can be integrated out from the very beginning. The $\mathbf{h}$-dependent part of the energy in the harmonic approximation is given by $\int d^{D} x \varkappa(\Delta \mathbf{h}+\boldsymbol{\beta})^{2} / 2$. An averaging over fluctuation of $\mathbf{h}$ and over disorder yields

$$
\left\langle\partial_{\alpha} \mathbf{h} \partial_{\alpha} \mathbf{h}\right\rangle=d_{c}\left(\frac{T}{\varkappa}+b\right) \int \frac{d^{D} \mathbf{q}}{(2 \pi)^{D}} \frac{1}{q^{2}} .
$$

For $D=2$ the integral diverges logarithmically. We will see below that $D=2$ is the lower critical dimension of the theory in the limit of infinite dimensionality of the embedding space $d \rightarrow \infty$. The special role of $D=2$ is not unexpected, since the theory that we are developing is based on an expansion near the ordered phase $(\xi>$ 0 ) and thus bears analogy with $\sigma$-models (cf. Ref. 55). Also, this role of $D=2$ can be foreseen already after a brief inspection of the free energy (16), (17), since both coupling constants $T / \kappa$ and $b$ have a dimensionality of $L^{D-2}$ and thus become dimensionless in 2D.

In a vicinity of the logarithmic dimension $D=2$, an $\mathrm{RG}$ formalism can be developed in the conventional way. 
Introducing an infrared cutoff $k$, making change of variables

$$
\tilde{\xi}^{2}=\xi^{2} k^{2-D},
$$

and using Eqs. (20) and (21), we find an RG equation that determines a flow of the stretching parameter $\xi$ with the spatial scale $k^{-1}$,

$$
\frac{d \tilde{\xi}^{2}}{d \Lambda}=(D-2) \tilde{\xi}^{2}-\frac{\tilde{d}_{c}}{4 \pi}\left(\frac{T}{\varkappa}+b\right)
$$

where $\Lambda=\ln \left(k_{m} / k\right)$ and

$$
\tilde{d}_{c}=\frac{d_{c}}{(4 \pi)^{(D-2) / 2} \Gamma(D / 2+1)} .
$$

Exactly at $D=2$, Eq. (23) simplifies to

$$
\frac{d \xi^{2}}{d \Lambda}=-\frac{d_{c}}{4 \pi}\left(\frac{T}{\varkappa}+b\right), \quad D=2 .
$$

The renormalization of $\xi$ is terminated by $k=1 / L$. The first and second terms in the r.h.s. of Eq. (25) describe contributions of dynamic fluctuations (flexural phonons) and of static deformations (ripples), respectively. Equation (25) predicts that $\xi$ becomes zero at a finite system size. In other words, a 2D membrane should be crumpled in the thermodynamic limit due to both dynamic and static deformations. While this conclusion is reminiscent of the Mermin-Wagner theorem $\underline{50}$ that forbids a long-range order in 2D systems, it turns out to be wrong (which is a manifestation of the fact that the MerminWagner theorem is not applicable to the problem under consideration ${ }^{55}$ ). Specifically, inclusion of anharmonicity leads to renormalization of $\varkappa$ and $b$ and restores the ordered (flat) phase in 2D for a finite dimensionality of the embedding space, $d<\infty$.

We are now going to derive RG equations describing the renormalization of elastic constants and disorder. We will see that at sufficiently large spatial scales, $\mu$ and $\lambda$ are screened to values independent on the bare ones. The effective theory at such scales is thus determined by three running couplings: the stretching parameter ("field renormalization") $\xi$, the bending rigidity ("stiffness") $\varkappa$ and the effective disorder strength $b$. We start from a discussion of renormalization of $\varkappa$ in the clean case $(b=$ 0 ) in Sec.IIIB and then derive the full set of RG equation for disordered membrane in Sec. IIIC.

\section{B. Renormalization of the bending rigidity in the clean case}

We start from the clean-membrane free energy $F(\tilde{\mathbf{u}}, \mathbf{h})$. Since the theory is quadratic with respect to longitudinal modes $\mathbf{u}$, they can be integrated out 52,65 The stretching parameter $\xi$ enters $F(\tilde{\mathbf{u}}, \mathbf{h})$ via $\tilde{\mathbf{u}}=\xi \mathbf{u}$ only, and evidently drops out after changing variables $\mathbf{u} \rightarrow \xi \mathbf{u}$ in the functional integral over $\mathbf{u}$. Hence, we arrive at the free energy that depends on $\mathbf{h}$ vectors only: $\mathfrak{6}^{65}$

$$
\begin{aligned}
\frac{F}{T} & =\frac{\varkappa}{2 T} \int(d k) k^{4}\left|\mathbf{h}_{\mathbf{k}}\right|^{2} \\
& +\frac{1}{4 d_{c}} \int\left(d k d k^{\prime} d q\right) R_{\mathbf{q}}\left(\mathbf{k}, \mathbf{k}^{\prime}\right)\left(\mathbf{h}_{\mathbf{k}+\mathbf{q}} \mathbf{h}_{-\mathbf{k}}\right)\left(\mathbf{h}_{-\mathbf{k}^{\prime}-\mathbf{q}} \mathbf{h}_{\mathbf{k}^{\prime}}\right) .
\end{aligned}
$$

Here and below we use a short-hand notation $(d k)=$ $d^{D} \mathbf{k} /(2 \pi)^{D}$. The kernel of quartic interaction between transverse modes (see Fig. 2) can be cast in the form (see Appendix B)

$$
R_{\mathbf{q}}\left(\mathbf{k}, \mathbf{k}^{\prime}\right)=\mathcal{N} \frac{\mathbf{k}_{\perp}^{2} \mathbf{k}_{\perp}^{\prime 2}}{D-1}+\mathcal{M}\left[\left(\mathbf{k}_{\perp} \mathbf{k}_{\perp}^{\prime}\right)^{2}-\frac{\mathbf{k}_{\perp}^{2} \mathbf{k}_{\perp}^{\prime 2}}{D-1}\right] .
$$

Here

$$
\mathbf{k}_{\perp}=\hat{P} \mathbf{k}=\mathbf{k}-\frac{\mathbf{q}(\mathbf{k q})}{q^{2}}
$$

where $\hat{P}$ is the projection operator related to the transferred momentum q,

$$
P_{\alpha \beta}=\delta_{\alpha \beta}-q_{\alpha} q_{\beta} / q^{2},
$$

and

$$
\mathcal{N}=\frac{\mu(2 \mu+D \lambda)}{(2 \mu+\lambda) T}, \quad \mathcal{M}=\frac{\mu}{T} .
$$

Hence, for a generic dimensionality $D$ of the membrane, there are two coupling constants, $\mathcal{N}$ and $\mathcal{M}$, controlling the interaction strength. An important exception is the case $D=2$ where the constant $\mathcal{M}$ drops out from the theory because the term in the square brackets in Eq. (27) turns to zero. Hence, for $D=2$ the interaction reads

$$
R_{\mathbf{q}}^{D=2}\left(\mathbf{k}, \mathbf{k}^{\prime}\right)=\frac{2 \mu(\mu+\lambda)}{(2 \mu+\lambda)} \frac{[\mathbf{k} \times \mathbf{q}]^{2}}{q^{2}} \frac{\left[\mathbf{k}^{\prime} \times \mathbf{q}\right]^{2}}{q^{2}} .
$$

The bare propagator (which is exact in the absence of interaction, $\mathcal{N}=\mathcal{M}=0$ ) is given by

$$
\left\langle h_{\mathbf{k}}^{\alpha} h_{-\mathbf{k}^{\prime}}^{\beta}\right\rangle=(2 \pi)^{D} \delta\left(\mathbf{k}-\mathbf{k}^{\prime}\right) \delta_{\alpha \beta} G_{\mathbf{k}}^{0},
$$

where

$$
G_{\mathbf{k}}^{0}=\frac{T}{\varkappa k^{4}} .
$$

The interaction coupling constants get screened in analogy with conventional charges in a media with a finite polarizability. Evaluating the polarization operator to the one-loop order [which is nothing but the random phase approximation (RPA)], we find the screened couplings:

$$
\begin{aligned}
\mathcal{N}_{\mathbf{q}} & =\frac{\mathcal{N}}{1+\mathcal{N}(D+1) \Pi_{\mathbf{q}}}, \\
\mathcal{M}_{\mathbf{q}} & =\frac{\mathcal{M}}{1+2 \mathcal{M} \Pi_{\mathbf{q}}}
\end{aligned}
$$



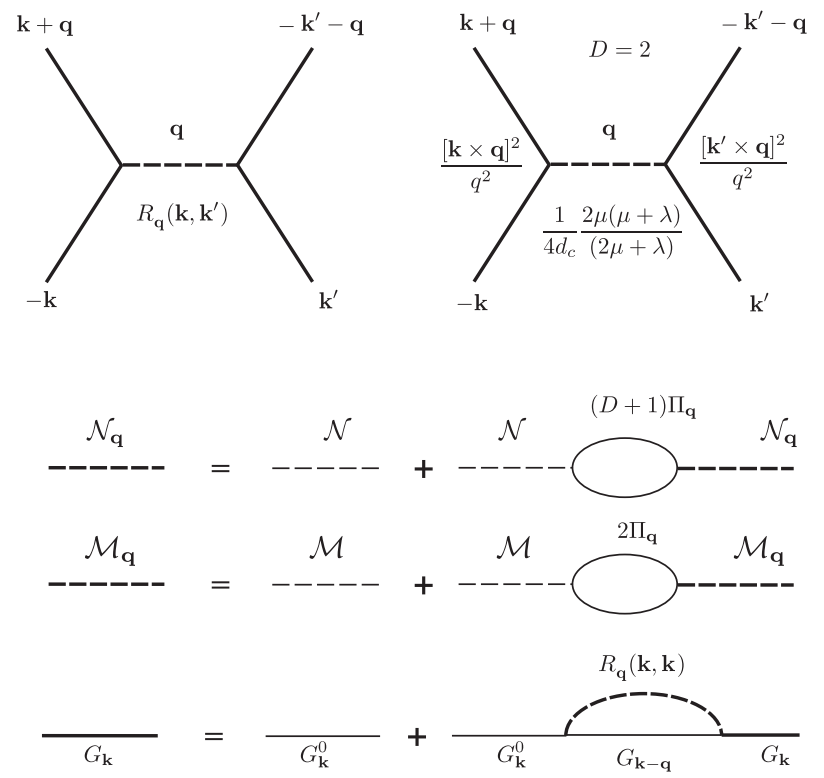

FIG. 2: Top: $h^{4}-$ interaction vertex for generic $D$ (left) and for $D=2$ (right). Bottom: One-loop diagrams describing renormalization of the interaction and of the propagator in a clean membrane.

Thus, the coupling constants $\mathcal{N}$ and $\mathcal{M}$ become $\mathbf{q}-$ dependent and are screened independently of each other. There is, however, an invariant subspace of the elastic coefficients,

$$
\lambda=-\frac{2 \mu}{D+2}
$$

where the coupling constants stay equal up to a numerical coefficient:

$$
\mathcal{N}_{\mathbf{q}}=\frac{2 \mathcal{M}_{\mathbf{q}}}{D+1}
$$

The polarization operator $\Pi_{\mathbf{q}}$ reads

$$
\Pi_{\mathbf{q}}=\frac{1}{D^{2}-1} \int(d k) k_{\perp}^{4} G_{\mathbf{k}}^{0} G_{\mathbf{q}-\mathbf{k}}^{0}
$$

Using (32), we get

$$
\Pi_{\mathbf{q}}=A_{D} \frac{T^{2}}{\varkappa^{2} q^{4-D}}
$$

where

$$
A_{D}=\frac{\Gamma\left(\frac{D}{2}\right) \Gamma\left(\frac{4-D}{2}\right)}{\pi^{(D-1) / 2} 2^{2 D+1} \Gamma\left(\frac{D+1}{2}\right)} .
$$

For $D=2$, we get $A_{2}=1 / 16 \pi$.

It follows from Eq. (38) that for any $D<4$ the polarization operator increases according to a power law with decreasing $q$. Hence, as seen from Eqs. (33) and (34), for sufficiently small $q$ couplings $\mathcal{N}$ and $\mathcal{M}$ become independent of their bare values and inversely proportional to the polarization operator:

$$
\mathcal{N}_{\mathbf{q}} \approx \frac{1}{(D+1) \Pi_{\mathbf{q}}}, \quad \mathcal{M}_{\mathbf{q}} \approx \frac{1}{2 \Pi_{\mathbf{q}}} .
$$

For the invariant subspace (35), Eqs. (40) hold for $q \ll$ $q^{*}$, where

$$
q^{*} \sim\left(\frac{\mu T}{\varkappa^{2}}\right)^{1 /(4-D)}
$$

is the inverse Ginzburg length which separates the normal region $\left(q>q^{*}\right)$ from the critical one $\left(q<q^{*}\right)$. For the case when bare couplings $\mathcal{N}$ and $\mathcal{M}$ are essentially different, there are two different scales $q_{\mathcal{N}}^{*}$ and $q_{\mathcal{M}}^{*}$ at which they become screened according to Eq. (40). For simplicity, we will assume that the bare values are of the same order, $\mathcal{N} \sim \mathcal{M}$, so that $q_{\mathcal{N}}^{*} \sim q_{\mathcal{M}}^{*} \sim q^{*}$. For a $2 \mathrm{D}$ membrane this question does not even arise, since the coupling $\mathcal{M}$ is simply absent.

We are now ready to evaluate the renormalization of the bending rigidity by (screened) interaction. The renormalized propagator of the $h$-field is given by

$$
G_{\mathbf{k}}=\frac{T}{\varkappa k^{4}+\Sigma_{\mathbf{k}}}
$$

where the one-loop self-energy reads

$$
\Sigma_{\mathbf{k}}=\frac{2 T}{d_{c}} \int(d q) \frac{\mathcal{N}_{\mathbf{q}}+(D-2) \mathcal{M}_{\mathbf{q}}}{D-1} k_{\perp}^{4} G^{0}(\mathbf{k}-\mathbf{q}) .
$$

For $q \ll q^{*}$, the effective screened interaction $\left[\mathcal{N}_{\mathbf{q}}+(D-\right.$ 2) $\left.\mathcal{M}_{\mathbf{q}}\right] /(D-1)$ that enters Eq. (43) becomes

$$
\frac{\mathcal{N}_{\mathbf{q}}+(D-2) \mathcal{M}_{\mathbf{q}}}{D-1} \approx \frac{D}{2(D+1) \Pi_{\mathbf{q}}} .
$$

Let us now substitute Eq. (44) into Eq. (43) and consider the behavior of $\Sigma_{\mathbf{k}}$ at low momenta $k \ll q^{*}$. Using Eq. (38) for the polarisation operator, we find that the integral in Eq. (43) scales as $k^{4} \ln (1 / k)$. This implies a logarithmic renormalization of the bending rigidity:

$$
\begin{aligned}
\delta \varkappa(\mathbf{k}) & \simeq \frac{D \varkappa}{d_{c}(D+1) A_{D}} \int_{0}^{q^{*}}(d q) \frac{k_{\perp}^{4} q^{4-D}}{k^{4}|\mathbf{k}-\mathbf{q}|^{4}} \\
& \simeq \eta \varkappa \ln \left(\frac{q^{*}}{k}\right),
\end{aligned}
$$

where we neglected the contribution from $q>q^{*}$ which is convergent for $k \rightarrow 0$ and is therefore small. The constant $\eta$ can be expressed as

$$
\eta=\frac{1}{d_{c}} \frac{D}{D+1}\left[\frac{B\left(D, \eta_{0}\right) \eta_{0}}{A\left(D, \eta_{0}\right)}\right]_{\eta_{0} \rightarrow 0},
$$

with integrals $A(D, \eta)$ and $B(D, \eta)$ as defined in Appendix The functions $A(D, \eta)$ and $B(D, \eta)$ will be used 
below for comparison with $\operatorname{SCSA} .65$ Note that $A(D, 0)=$ $A_{D}$.

For a sufficiently large spatial scale $k^{-1}$ the correction (45) ceases to be small and gets promoted to a RG equation,

$$
\frac{d \varkappa}{d \Lambda}=\eta \varkappa
$$

where $\Lambda=\ln \left(q^{*} / k\right)$. We thus see that $\eta$ is the anomalous dimension of the bending rigidity. Using Eqs. (C1) and (C2), we find

$$
\eta=\frac{D(D-1) 2^{D}}{\sqrt{\pi} d_{c}} \frac{\Gamma\left(\frac{D+1}{2}\right)}{\Gamma\left(\frac{D}{2}\right) \Gamma\left(2-\frac{D}{2}\right) \Gamma\left(2+\frac{D}{2}\right)} .
$$

It is worth pointing out that, at variance with Eq. 21), the integral (45) is always logarithmic (i.e., also away from $D=2$ ). Thus, Eq. (48) is applicable for any $D$ as well. The only condition of validity of this equation is $d_{c} \gg 1$, which implies that $\eta \ll 1$. The equation simplifies for $D=2$ and $D=4-\epsilon$, yielding

$$
\eta \simeq\left\{\begin{aligned}
2 / d_{c}, & \text { for } D=2, \\
12 \epsilon / d_{c}, & \text { for } D=4-\epsilon .
\end{aligned}\right.
$$

[Since $d_{c} \gg 1$, the second line in this equation agrees with Eq. (5) obtained by $\epsilon$-expansion.] For generic values of $d$ and $D$, the exponent $\eta$ is not small. To find it, one would have to take into account higher-loop contributions to RG equations. Such a calculation does not appear feasible because of the absence of a small parameter. On the other hand, one may develop a self-consistent extension of the one-loop theory by inserting renormalized Green functions into the one-loop diagrams, which amounts to the replacement ${ }^{65}$ of $G^{0}$ with $G$ in Eqs. (37) and (43). Corresponding calculations yield a self-consistency equation for $\eta$

$$
1=\frac{1}{d_{c}} \frac{D}{D+1} \frac{B(D, \eta)}{A(D, \eta)},
$$

which was derived in Ref. 65. This equation can be obtained from Eq. (46) by replacing $\eta_{0}$ with $\eta$. Although such a procedure is not controlled parametrically for physical membranes $(d=3, D=2)$, it gives in this case a value $\eta \approx 0.821$ which turns out to be in a reasonable agreement ${ }^{91}$ with numerical values $\eta=0.60 \pm 0.10$ and $\eta=0.72 \pm 0.04$ (see Ref. 63 and Ref. 68, respectively).

\section{Renormalization group for disordered membrane}

Now we include in the consideration the random curvature disorder which modifies only the first term in Eq. (26). In the coordinate representation, this term becomes

$$
F_{0}=\frac{\varkappa}{2} \int d^{D} \mathbf{x}(\Delta \mathbf{h}+\boldsymbol{\beta})^{2}
$$

where $\boldsymbol{\beta}=\boldsymbol{\beta}(\mathrm{x})$ is a random vector with Gaussian distribution (17). Disorder averaging can be performed with making use of the replica trick. To this end, we introduce $N$ replicas of the field $\mathbf{h}$ (i.e., make a replacement $\mathbf{h} \rightarrow \mathbf{h}^{n}$ with a replica index $\left.n=1, \ldots, N\right)$ and replicate the free energy $F$ :

$$
F^{\mathrm{rep}}=F_{0}^{\mathrm{rep}}+F_{1}^{\mathrm{rep}}
$$

where

$$
F_{0}^{\mathrm{rep}}=\sum_{n=1}^{n=N} \frac{\varkappa}{2} \int(d k) k^{4}\left|\mathbf{h}_{\mathbf{k}}^{n}+\boldsymbol{\beta}_{\mathbf{k}}\right|^{2}
$$

and

$$
\begin{aligned}
& F_{1}^{\mathrm{rep}}=\sum_{n=1}^{n=N} \frac{1}{4 d_{c}} \\
& \times \int\left(d k d k^{\prime} d q\right) R_{\mathbf{q}}\left(\mathbf{k}, \mathbf{k}^{\prime}\right)\left(\mathbf{h}_{\mathbf{k}+\mathbf{q}}^{n} \mathbf{h}_{-\mathbf{k}}^{n}\right)\left(\mathbf{h}_{-\mathbf{k}^{\prime}-\mathbf{q}}^{n} \mathbf{h}_{\mathbf{k}^{\prime}}^{n}\right) .
\end{aligned}
$$

Next, we average $\exp \left(-F_{\text {rep }} / T\right)$ with $P(\boldsymbol{\beta})$, thus arriving at the following effective action:

$$
F_{\text {eff }}=\frac{1}{2} \sum_{n, m} \int(d k) \varkappa^{n m} k^{4} \mathbf{h}_{\mathbf{k}}^{n} \mathbf{h}_{-\mathbf{k}}^{m}+F_{1}^{\mathrm{rep}},
$$

where we have introduced a replica-space matrix $\hat{\varkappa}$ with elements $\varkappa^{n m}$ given by

$$
\hat{\varkappa}=\varkappa-\frac{b \varkappa^{2}}{T+b \varkappa N} \hat{J} .
$$

Here $\hat{J}$ is a matrix with all elements equal to unity: $J^{n m}=1$. The bare propagator thus becomes a matrix in the replica space:

$$
\hat{G}_{\mathbf{k}}^{0}=\frac{T \hat{\varkappa}^{-1}}{k^{4}}=\frac{T}{\varkappa k^{4}}(1+f \hat{J}),
$$

where

$$
f=\frac{b \varkappa}{T}
$$

is the dimensionless parameter characterising a ratio between the bare disorder, $b$, and the bare dynamical fluctuations, $T / \kappa$.

The polarization operator also becomes a replica-space matrix. In the one-loop order (i.e., within RPA), its elements read

$$
\begin{aligned}
\Pi_{\mathbf{q}}^{n m} & =\frac{1}{D^{2}-1} \int(d k) k_{\perp}^{4} G_{\mathbf{k}}^{0, n m} G_{\mathbf{q}-\mathbf{k}}^{0, n m} \\
& =\frac{T^{2}}{D^{2}-1} \int(d k) k_{\perp}^{4} \frac{\left(\hat{\varkappa}^{-1}\right)^{n m}}{k^{4}} \frac{\left(\hat{\varkappa}^{-1}\right)^{n m}}{|\mathbf{q}-\mathbf{k}|^{4}} .
\end{aligned}
$$

Using the property $\left(\delta^{n m}+f J^{n m}\right)^{2}=(1+2 f) \delta^{n m}+$ $f^{2} J^{n m}$, we find

$$
\hat{\Pi}_{\mathbf{q}}=A_{D} \frac{T^{2}}{\varkappa^{2} q^{4-D}}\left(1+2 f+f^{2} \hat{J}\right) .
$$


Substituting Eq. (60) into Eqs. (33) and (34), we obtain the screened couplings $\hat{\mathcal{N}}_{\mathbf{q}}$ and $\hat{\mathcal{M}}_{\mathbf{q}}$ as matrices in the replica space. The Ginzburg scale $q^{*}$ is now affected by the disorder that enters the polarization operator (60):

$$
q^{*} \sim\left[\frac{\mu T(1+2 f)}{\varkappa^{2}}\right]^{1 /(4-D)} .
$$

It is worth noting that for strong disorder or low temperatures $(f \gg 1) q^{*} \sim(\mu b / \kappa)^{1 /(4-D)}$ is independent of temperature, while for weak disorder $(f \ll 1)$ we recover Eq. (41), $q^{*} \propto T^{1 / 2}$.

Let us now calculate the self-energy determining the renormalization of the bending rigidity. In similarity with Eq. (44), the effective interaction $\hat{U}$ with matrix elements $U_{n m}$ (see Fig. 3) is expressed for $q \ll q^{*}$ in terms of the polarization operator,

$$
\begin{aligned}
\hat{U} & =\frac{D \hat{\Pi}_{\mathbf{q}}^{-1}}{2(D+1)} \\
& =\frac{D}{2(D+1)} \frac{\varkappa^{2} q^{4-D}}{T^{2} A_{D}} \frac{1+2 f+f^{2} N-f^{2} \hat{J}}{(1+2 f)\left(1+2 f+f^{2} N\right)} .
\end{aligned}
$$

The replica generalization of Eq. (43) for self-energy thus takes the form

$$
\Sigma_{\mathbf{k}}^{n m}=\frac{2 T}{d_{c}} \int(d q) k_{\perp}^{4} \frac{D\left(\Pi_{\mathbf{q}}^{-1}\right)^{n m}}{2(D+1)} G_{\mathbf{k}-\mathbf{q}}^{0, n m} .
$$

Substituting here the Green function, Eq. (57), using the property

$$
\begin{aligned}
& {\left[1+2 f+f^{2} N-f^{2} \hat{J}\right]^{n m}(1+f \hat{J})^{n m}} \\
& =\left[1+3 f+f^{2}(N+1)+f^{3} N-f^{3} \hat{J}\right]^{n m},
\end{aligned}
$$

and taking the limit $N \rightarrow 0$, we finally arrive at the matrix equation which governs the renormalization of $\hat{\varkappa}$ in the presence of disorder:

$$
\frac{d \hat{\varkappa}}{d \Lambda}=\eta \varkappa \frac{1+3 f+f^{2}-f^{3} \hat{J}}{(1+2 f)^{2}}
$$

where $\eta$ is given by Eq. (46). Substituting Eq. (56) into l.h.s. of Eq. (64), and separating terms proportional to $\hat{J}$ from scalar ones, we find two equations describing renormalization of the bending rigidity and of the disorder strength:

$$
\begin{aligned}
\frac{d \varkappa}{d \Lambda} & =\eta \varkappa \frac{1+3 f+f^{2}}{(1+2 f)^{2}}, \\
\frac{d(\varkappa f)}{d \Lambda} & =\eta \varkappa \frac{f^{3}}{(1+2 f)^{2}} .
\end{aligned}
$$

Equations (23), (65), (66) represent the full set of RG equations which determine the behavior of the system for $q \ll q^{*}$ [the Ginzburg scale $q^{*}$ is defined here with the bare disorder strength $f_{0}$ in Eq. [61)]. To analyse
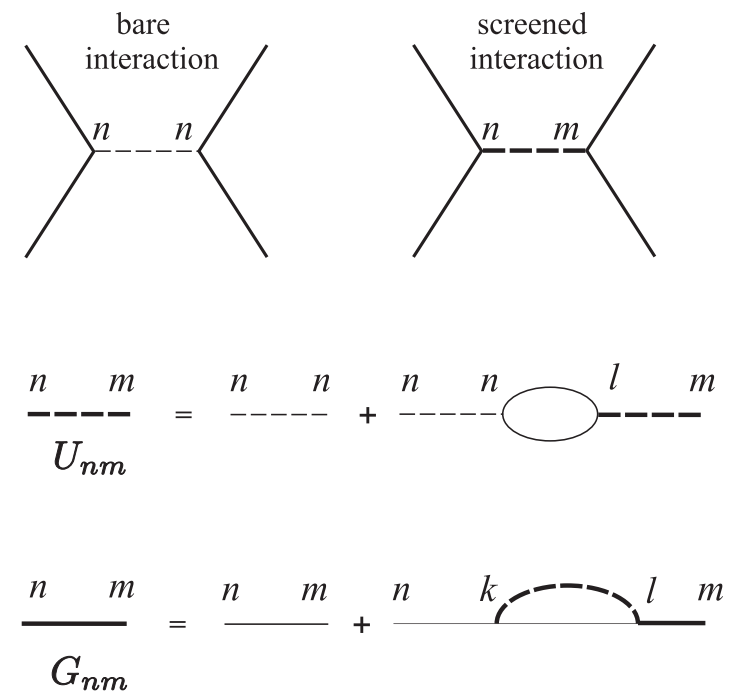

FIG. 3: Replica-index structure of the $h^{4}$-interaction and of the one-loop diagrams for a disordered membrane.

the fixed points and the scaling flow, we rewrite them in terms of appropriate dimensionless couplings. To this end, we introduce a rescaled bending rigidity,

$$
\tilde{\varkappa}=\varkappa \tilde{\xi}^{2}=\varkappa \xi^{2} k^{2-D} \text {. }
$$

The meaning of Eq. 67 is twofold. First, the ratio $\tilde{\varkappa} / T$ is made dimensionless (also for a dimensionality deviating from $D=2$ ). Second, it takes into account the "compression" of the membrane controlled by the factor $\xi$ in Eq. (18), which can be viewed as an analog of the order-parameter field renormalization in the conventional $\sigma$-model RG. Expressing further the flow of $f$ from Eqs. (65) and (66), we cast the RG equation into the following form:

$$
\begin{aligned}
\frac{d \xi^{2}}{d \Lambda} & =-\frac{\tilde{d}_{c}}{4 \pi} \frac{T}{\tilde{\varkappa}}(1+f) \xi^{2} \\
\frac{d \tilde{\varkappa}}{d \Lambda} & =\tilde{\varkappa}\left[D-2+\eta \frac{1+3 f+f^{2}}{(1+2 f)^{2}}\right]-\frac{\tilde{d}_{c} T}{4 \pi}(1+f) \\
\frac{d f}{d \Lambda} & =-\eta \frac{f(1+3 f)}{(1+2 f)^{2}}
\end{aligned}
$$

It is worth discussing a structure of the derived RG equations. Equations (69) and (70) describe evolution of two dimensionless couplings of the theory: the inverse dimensionless bending rigidity $T / \tilde{\kappa}$ and the dimensionless disorder $\tilde{b}=f T / \tilde{\kappa}$. The solution of one-loop equations is simplified by the fact that the equation for $f$ (ratio of disorder to the inverse bending rigidity), Eq. (70), decouples and thus can be straightforwardly integrated. After this, Eq. (69) for $\tilde{\varkappa}$ can be solved. Finally, Eq. (68) is analogous to the wave-function renormalization equation of the $\sigma$-model RG. It is a "slave" equation which can be solved at the last stage.

We emphasize that the set of the RG equations (68), (69), and (70) strongly differs from the RG equa- 
tions $161,62,66$ previously derived in dimensionality $D=$ $4-\epsilon$. Apart from the dimensionality (that allows us to employ the RG approach to physical $D=2$ graphene samples), the crucial difference is in the role of screening near two dimensions. In our case, the screening of anharmonic couplings eliminates the separate renormalization of elastic constants $\lambda$ and $\mu$; they now only determine the starting point of the RG, $q^{*}$, Eq. (61). For the same reason, only the out-of-plane (curvature) short-range disorder survives the renormalization, see discussion of the in-plane disorder in Sec. IIID below.

One can further simplify Eqs. (68) and (69) by introducing a dimensionless coupling

$$
K=\frac{4 \pi \eta \tilde{\varkappa}}{\tilde{d}_{c} T(1+f)}
$$

[for $D=2$ this transformation takes the form $K=$ $\left.\left(8 \pi / d_{c}^{2} T\right) \tilde{\varkappa} /(1+f)\right]$. We also change the variable $\Lambda$ to

$$
z=\eta \Lambda,
$$

thus arriving at the following $\mathrm{RG}$ equations:

$$
\begin{aligned}
\frac{d \xi^{2}}{d z} & =-\frac{\xi^{2}}{K} \\
\frac{d K}{d z} & =K\left[\epsilon_{2}+\frac{1+5 f+7 f^{2}+f^{3}}{(1+f)(1+2 f)^{2}}\right]-1, \\
\frac{d f}{d z} & =-\frac{f(1+3 f)}{(1+2 f)^{2}} .
\end{aligned}
$$

Here

$$
\epsilon_{2}=\frac{D-2}{\eta} \simeq \frac{(D-2) d_{c}}{2} .
$$

Before proceeding to a detailed analysis of these RG equations, we briefly discuss the effect of in-plane disorder.

\section{In-plane disorder}

In most of this paper, we explore the problem in the presence of out-of-plane ("random curvature") disorder. In this subsection we briefly analyse the in-plane disorder and show that, with an exception of the case of topological defects (disclinations), it can be safely neglected. We restrict ourselves to the case $D=2$. Let us consider an impurity-induced isotropic in-plane disorder that leads 62 to the following modification of the free energy (26):

$$
\begin{aligned}
F & =\frac{\varkappa}{2} \int(d k) k^{4}\left|\mathbf{h}_{\mathbf{k}}\right|^{2} \\
& +\frac{\mathcal{N}}{4 d_{c}} \int(d q)\left|\int(d k) \frac{(\mathbf{k} \times \mathbf{q})^{2}}{q^{2}} \mathbf{h}_{\mathbf{k}+\mathbf{q}} \mathbf{h}_{-\mathbf{k}}+c_{\mathbf{q}}\right|^{2} .
\end{aligned}
$$

Here $c_{\mathbf{q}}$ is the Fourier transform of the random quenched field $c(\mathbf{x})$ with the Gaussian distribution

$$
P[c(\mathbf{x})] \propto \exp \left(-\frac{1}{2 \sigma} \int d \mathbf{x} c^{2}(\mathbf{x})\right),
$$

and $\sigma$ is the effective disorder strength. Replicating the field $\mathbf{h}$ and averaging over disorder, we find that the bare interaction $U_{n m}^{0}$ (see Fig. 3) acquires now off-diagonal elements in the replica space:

$$
U_{n m}^{0}=\mathcal{N} \delta_{n m}-\mathcal{N}^{2} \sigma \hat{J} .
$$

Next, we find the screened interaction by solving the equation $\hat{U}=\hat{U}_{0}-\hat{U}_{0} \Pi \hat{U}$ (see Fig. 33), which yields

$$
\hat{U}=\left(1+\hat{U}_{0} \Pi\right)^{-1} \hat{U}_{0}=\left(1+\Pi^{-1} \hat{U}_{0}^{-1}\right)^{-1} \Pi^{-1} .
$$

For $q \ll q^{*}$, the polarization operator grows as $1 / q^{2}$, so that one can neglect the term $\Pi^{-1} \hat{U}_{0}^{-1} \sim q^{2}$. As a result, both the elastic constant $\mathcal{N}$ and the disorder strength $\sigma$ drop out from the effective interaction:

$$
\hat{U} \rightarrow \Pi^{-1}, \text { for } q \ll q^{*} .
$$

We thus conclude that, in the critical region $q \ll q^{*}$, the in-plane random potential becomes irrelevant and does not affect critical behavior of $\varkappa$.

This conclusion should be contrasted with that of Ref. 64 where it was argued that an arbitrarily weak inplane impurity disorder destroys the flat phase. Specifically, it was found that the membrane is destroyed for $\sigma>\sigma_{c}^{(0)}$, where $1 / \sigma_{c}^{(0)}$ is strongly divergent in the infrared limit: $1 / \sigma_{c}^{(0)} \propto 1 / q_{\mathrm{IR}}^{2}$. Here, $q_{\mathrm{IR}}$ is the infrared cutoff momentum (inverse system size, $q_{\mathrm{IR}} \sim 1 / L$ ). One can demonstrate that in fact screening cures this divergency (cf. Ref. 67) and leads to a replacement of $q_{\mathrm{IR}}$ with $q^{*}$, thus yielding a finite value $\sigma_{c} \propto T / \mu$. A not too strong in-plane disorder, $\sigma_{0}<\sigma_{c}$ will thus simply lead to ultraviolet corrections to the bare parameters (out-ofplane disorder $b_{0}$ and bending rigidity $\varkappa_{0}$ ) of our theory. Specifically, a natural expectation is that the inplane disorder would enhance $b_{0}$ and reduce $\varkappa_{0}$. In other words, the in-plane disorder is RG-irrelevant at length scales $\gtrsim 1 / q_{*}$ but its contribution on shorter (atomic) scales can affect the starting point of RG.

The above result on irrelevance of the in-plane disorder was based on an assumption of a finite-range disorder $c(\mathbf{x})$, which means that the disorder strength $\sigma_{\mathbf{q}}$ remains finite in the limit $\mathbf{q} \rightarrow 0$. It is easy to see that this assumption can be in fact weakened: it is sufficient to require that the singularity of $\sigma_{\mathbf{q}}$ at $\mathbf{q} \rightarrow 0$ (if present) is not stronger than $1 / q^{2-\alpha}$ with $\alpha>0$. This corresponds to spatial correlations with a power-law decay, $\langle c(0) c(\mathbf{x})\rangle \propto x^{-\alpha}$. In this case, inverting Eq. (78) and taking the limit $N \rightarrow 0$, we find

$$
\hat{U}_{0}^{-1}(N \rightarrow 0)=\frac{1}{\mathcal{N}}+\sigma_{q} \hat{J} .
$$

Since $\sigma_{q} \Pi^{-1} \rightarrow q^{\alpha}$ for $q \rightarrow 0$, we conclude that $\hat{U}_{0}^{-1} \Pi^{-1}$ vanishes at small $q$, so that the derivation of (80) retains its validity. Only for $\alpha \rightarrow 0$ (which corresponds to logarithmic real-space correlations) we get $\sigma_{q} \Pi^{-1} \rightarrow$ const for $q \rightarrow 0$. Such a disorder is RG-marginal and thus 
will influence the flow of other couplings. Physically, the $1 / q^{2}$ in-plane disorder corresponds to random topological defects - disclinations (see Ref. 67 for a discussion of different types of long-range disorder and their treatment within the SCSA). A detailed analysis of this type of inplane disorder is outside of the scope of this paper and will be presented elsewhere.

\section{ANALYSIS OF RG EQUATIONS: CRUMPLING TRANSITION}

In this section we analyze the $\mathrm{RG}$ equations derived above for clean and disordered cases.

\section{A. Clean membrane}

In the absence of disorder $(f=0)$ and for $D=2$, Eqs. (73) and (74) take the form

$$
\begin{aligned}
& \frac{d \xi^{2}}{d z}=-\frac{\xi^{2}}{K} \\
& \frac{d K}{d z}=K-1 .
\end{aligned}
$$

Here we have taken into account that $\tilde{\xi}=\xi$ for $D=2$. The initial condition for Eq. (82) is 92

$$
\xi_{0}=1, \quad \text { for } z=0 \text {. }
$$

From Eqs. (82) and (83) we conclude that there exists an unstable fixed point

$$
K_{\mathrm{cr}}=1
$$

or, equivalently,

$$
\varkappa_{\mathrm{cr}}=\frac{d_{c}^{2} T}{8 \pi} .
$$

Indeed, assuming that the starting value of the bending rigidity, $\varkappa_{0}=\tilde{\varkappa}_{z=0}=\varkappa_{z=0}$, exceeds the critical value, $\varkappa_{0}>\varkappa_{\mathrm{cr}}$, we find from Eqs. (82) and (83)

$$
\xi^{2}=\frac{\varkappa_{\mathrm{cr}} e^{-z}+\varkappa_{0}-\varkappa_{\mathrm{cr}}}{\varkappa_{0}}
$$

and, consequently,

$$
\xi_{z=\infty}^{2}=\frac{\varkappa_{0}-\varkappa_{\mathrm{cr}}}{\varkappa_{0}}
$$

Therefore, above the critical point membrane remains in the flat phase in the course of renormalization. On the other hand, one can easily check that below the critical point (for $\varkappa_{0}<\varkappa_{\mathrm{cr}}$ ), the membrane shrinks to the crumpled phase, $\xi=0$, at a finite scale

$$
L \sim q^{-1}=\frac{1}{q^{*}}\left(\frac{\varkappa_{\mathrm{cr}}}{\varkappa_{\mathrm{cr}}-\varkappa_{0}}\right)^{d_{c} / 2} .
$$

Hence, the fixed point (86) separates the crumpled and flat phases 55

For a membrane dimensionality $D$ slightly deviating from 2, we get instead of (83)

$$
\frac{d K}{d z}=K\left(\epsilon_{2}+1\right)-1 .
$$

Equation (90) implies that the lower critical dimension for crumpling transition can be found from the condition $\epsilon_{2}=-1$, which yields

$$
D_{\mathrm{cr}}=2-\frac{2}{d_{c}}
$$

in agreement with previous studies $\stackrel{59}{.}$.

Exactly at the transition point, when $K=K_{\mathrm{cr}}=$ $1 /\left(1+\epsilon_{2}\right)$, the stretching factor $\xi$ decays with $L$ according to a power law:

$$
\xi \propto \frac{1}{L^{\tau}}
$$

where $\tau=(D-2+\eta) / 2$. In other words, the extension of the membrane $R$ in the embedding space scales with its "intrinsic" length $L$ as $R=L^{1-\tau}$. The exponent $\tau$ determines thus the fractal (Hausdorff) dimension $D_{H}$ of the membrane at criticality (defined by the relation $\left.R^{D_{H}} \sim L^{D}\right)$, yielding $D_{H}=D /(1-\tau)=2 D /(4-D-\eta)$.

Let us discuss in more detail geometric properties of the membrane, which are determined by the behavior of $\xi$. To this end, let us consider two points $\mathbf{r}_{1}$ and $\mathbf{r}_{2}$ on the membrane and the corresponding points $\mathbf{x}_{1}$ and $\mathbf{x}_{2}$ in the reference plane. According to Eq. (18) and to the $\mathrm{RG}$ procedure, we have a scaling relation

$$
\left\langle\left|\mathbf{r}_{1}-\mathbf{r}_{2}\right|^{2}\right\rangle \sim \xi_{\left|\mathbf{x}_{1}-\mathbf{x}_{2}\right|}^{2}\left(\mathbf{x}_{1}-\mathbf{x}_{2}\right)^{2},
$$

where $\xi_{x}$ is the value of the running renormalizationparameter $\xi$ at the RG spatial scale $x$. When $\xi_{x}$ drops down to a value substantially smaller than unity (say, to $1 / 2)$, the embedding-space distance $\left|\mathbf{r}_{1}-\mathbf{r}_{2}\right|$ between the points become essentially smaller than the intrinsic distance $\left|\mathbf{x}_{1}-\mathbf{x}_{2}\right|$. This indicates that a membrane of such size starts to show "folding" (i.e., strong spatial variation of the normal vector to the membrane in the embedding space).

Deep in the flat phase, the renormalization of $\xi$ at the whole interval of RG scales, $1 / q^{*}<L<\infty$, is relatively weak. This implies that the membrane does not fold. In other words, although the surface of a membrane is not exactly flat due to dynamical fluctuations (and also due to ripples in the disordered case, as discussed below), the spatial variation of the normal vector remains relatively small.

On the other hand, when $\varkappa_{0}$ approaches the critical value, Eq. (86) (i.e., when $\varkappa_{0}-\varkappa_{\mathrm{cr}} \ll \varkappa_{\mathrm{cr}}$ ), the membrane shows fractal folding at the broad interval of length scales. Specifically, as seen from Eq, (87), this folding becomes strong for $z \sim 1$, i.e. at the length scale $L \sim L_{1}$, 
where

$$
L_{1} \sim \frac{1}{q^{*}} e^{1 / \eta} .
$$

At a much larger scale,

$$
L_{2} \sim \frac{1}{q^{*}}\left(\frac{\varkappa_{\mathrm{cr}}}{\varkappa_{0}-\varkappa_{\mathrm{cr}}}\right)^{1 / \eta},
$$

$\exp (-z)$ becomes on the order of $\left(\varkappa_{0}-\varkappa_{\mathrm{cr}}\right) / \varkappa_{\mathrm{cr}}$ and $\xi$ saturates. Thus, the membrane has a fractal geometry in the interval $L_{1} \ll L \ll L_{2}$. At larger scales, $L \gg L_{2}$ the membrane flattens. Exactly at the transition, $L_{2}$ diverges and the membrane remains fractal at arbitrarily large scales.

\section{B. Disordered membrane}

\section{RG flow in disordered case}

Let us now consider the disordered case. From now on and till the end of the paper, we will assume that $D=2$ and, consequently, $\epsilon_{2}=0$. We see from Eq. (75) that $f$ monotonously decreases in course of renormalization. Dividing Eq. (74) by Eq. (75), we find an equation that determines variation of $K$ with $f$. Its solution yields the function $K(f)$ which can be written in the following form:

$$
K(f)=K_{\mathrm{cr}}(f)+\frac{(3 f+1)^{1 / 9} e^{-f / 3}}{f(1+f)} \delta .
$$

Here $K_{\mathrm{cr}}(f)$ is the critical curve in the $(K, f)$ plane which separates the crumpled and flat phases (see Fig. (4):

$$
K_{\mathrm{cr}}(f)=\frac{(3 f+1)^{1 / 9} e^{-f / 3}}{f(1+f)} \int_{0}^{f} d y \frac{(1+2 y)^{2}(1+y) e^{y / 3}}{(3 y+1)^{10 / 9}}
$$

The phase boundary has the following asymptotic behaviour at small and large $f$ :

$$
K_{\mathrm{cr}} \simeq\left\{\begin{aligned}
1+f^{2} / 3, & \text { for } f \rightarrow 0 \\
4(1-5 / f), & \text { for } f \rightarrow \infty .
\end{aligned}\right.
$$

For $f=0$ we recover Eq. (85) as expected. The parameter $\delta$ controlling the deviation of the RG flow line from the critical one depends on initial values of $f$ and $K$ in the following way:

$$
\delta=\frac{f_{0}\left(1+f_{0}\right)}{\left(3 f_{0}+1\right)^{1 / 9}} e^{f_{0} / 3}\left[K_{0}-K_{\mathrm{cr}}\left(f_{0}\right)\right] .
$$

The flat phase corresponds to $\delta>0$, while the crumpled phase to $\delta<0$.

The RG flow in the $(K, f)$ plane is illustrated in Fig. (44). One observes that in the flat phase the evolution of $K$ is non-monotonous. For $\delta \ll 1$ the minimum of $K$
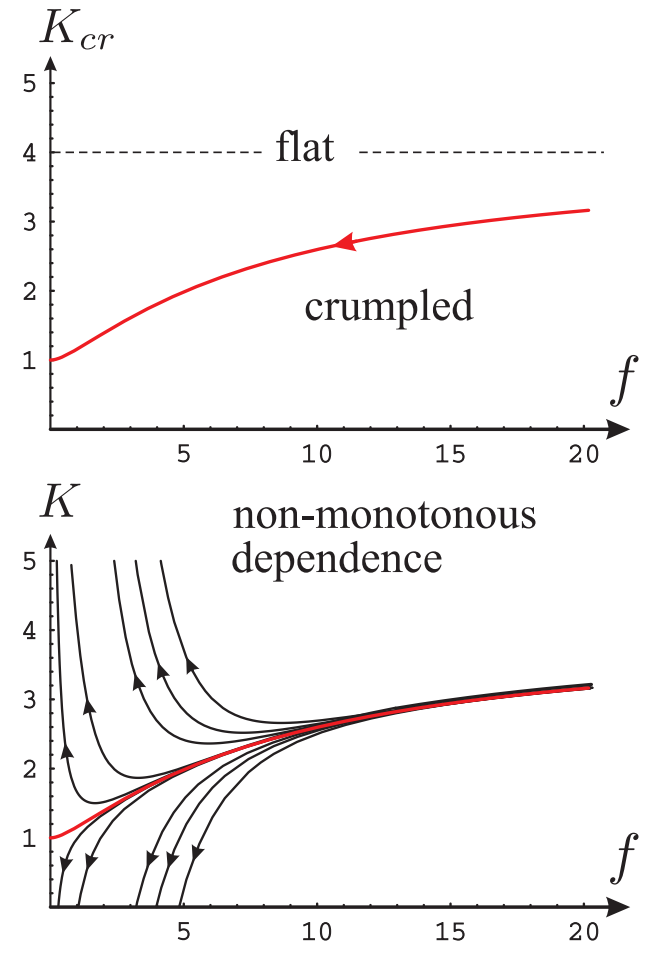

FIG. 4: Upper panel: Critical curve $(\delta=0)$ separating the crumpled and flat phases in the $K-f$ plane. Lower panel: RG flow lines in the $K-f$ plane for a disordered membrane for different values of $\delta(\delta=-200,-100,-50,-3,-0.4,0,1.2$, $7.5,50,100,200$ from bottom to top).

lies in the region of small $f$, where $K \approx 1+f^{2} / 3+\delta / f$. Minimization yields the position of the minimum:

$$
f_{\min } \approx(3 \delta / 2)^{1 / 3}, K_{\min } \approx 1+(3 \delta / 2)^{2 / 3}, \text { for } \delta \ll 1 .
$$

In the opposite case, $\delta \gg 1$, the minimum corresponds to large $f$, where $K \approx 4-20 / f+9 \delta \exp (-f / 3) /(3 f)^{17 / 9}$. We find with logarithmical precision

$$
f_{\min } \approx 3 \ln (\delta), K_{\min } \approx 4-20 / 3 \ln (\delta), \text { for } \delta \gg 1 .
$$

\section{Disorder-induced crumpling}

Let us fix initial value of $\varkappa_{0}=\tilde{\varkappa}_{z=0}=\varkappa_{z=0}$ and consider what happens with increasing disorder strength $b$. Since $\xi_{0}=1$, we have the following initial values of $K$ and $f$ :

$$
K_{0}=\frac{\varkappa_{0}}{\varkappa_{\mathrm{cr}}\left(1+f_{0}\right)}, \quad f_{0}=\frac{b_{0} \varkappa_{0}}{T},
$$

where $\varkappa_{\mathrm{cr}}$ is given by Eq. (86). The crumpling occurs when $K_{0}$ becomes smaller than $K_{\mathrm{cr}}\left(f_{0}\right)$. Hence, the critical curve in the $\left(\varkappa_{0}, b_{0}\right)$ plane is implicitly determined by equations $\varkappa_{0}=\varkappa_{\mathrm{cr}}\left(1+f_{0}\right) K_{\mathrm{cr}}\left(f_{0}\right)$ and $b_{0}=f_{0} T / \varkappa_{0}=$ $\left(8 \pi / d^{2}\right) f_{0} /\left(1+f_{0}\right) K_{\mathrm{cr}}\left(f_{0}\right)$, with $f_{0}$ varying in the interval 


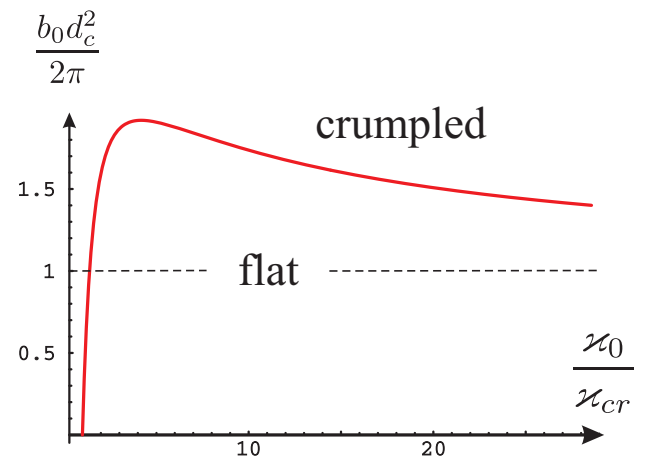

FIG. 5: Crumpling-transition phase diagram in the plane of initial parameters (bending rigidity $\varkappa_{0}$ and disorder $b_{0}$ ).

$(0, \infty)$. The dependence $b_{0}\left(\varkappa_{0}\right)$ found from these equations is plotted in Fig. (5). The asymptotic behaviour of the critical line reads

$$
b_{0}=\frac{2 \pi}{d_{c}^{2}}\left\{\begin{array}{cl}
4\left(\varkappa_{0}-\varkappa_{\mathrm{cr}}\right) / \varkappa_{\mathrm{cr}}, & \text { for } \varkappa_{0} \rightarrow \varkappa_{\mathrm{cr}} ; \\
1+19 \varkappa_{\mathrm{cr}} / 16 \varkappa_{0}, & \text { for } \varkappa_{0} \rightarrow \infty
\end{array}\right.
$$

We notice that the dependence is non-monotonous. In other words, there exists an optimal value of bare bending rigidity, $\varkappa_{0} \approx 4.2 \varkappa_{\mathrm{cr}}$, for which the membrane is most robust to disorder-induced crumpling. It is also worth stressing that the critical disorder saturates in the limit of infinite bending rigidity at the universal value $b_{0}=$ $2 \pi / d_{c}^{2}$.

\section{RIPPLING AND FOLDING OF MEMBRANE IN THE FLAT PHASE}

We have thus established the phase diagram of the crumpling transition in the plane of initial parameters, i.e., bending rigidity $\varkappa_{0}$ and disorder $b_{0}$. An important question that remains to be explored is the evolution of physical observables with the length scale. This will be done in the present section. We will, in particular, show that the disorder strength decreases beyond a certain length scale $L_{r}$.

After rescaling the disorder strength

$$
\tilde{b}=\frac{b}{\xi^{2}},
$$

one finds that the RG equations in the $(\tilde{\varkappa}, \tilde{b})$-plane are implicitly determined by the following equations

$$
\begin{aligned}
\frac{\tilde{\varkappa}}{\varkappa_{\mathrm{cr}}} & =(1+f) K(f), \\
\frac{\tilde{b} d_{c}^{2}}{2 \pi} & =\frac{4 f}{(1+f) K(f)},
\end{aligned}
$$

where $K(f)$ is given by Eq. (96) and $f$ varies in the interval $[0, \infty]$. For $\delta=0$ we reproduce the critical curve $b_{0}\left(\varkappa_{0}\right)$ separating the crumpled and flat phases, see

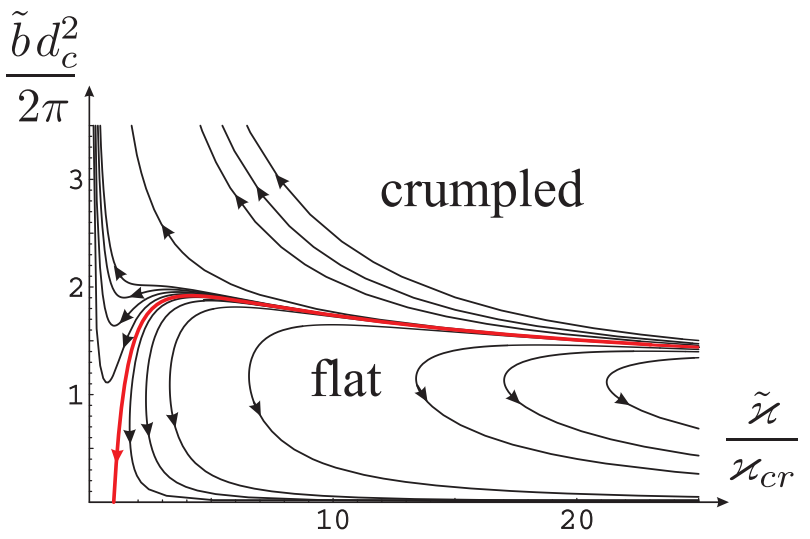

FIG. 6: RG flow in the $(\tilde{\varkappa}, \tilde{b})-$ plane for $\delta=-200,-100$, $50,-3,-0.4,-0.3,-0.2,-0.1,0,0.1,0.3,1.2,7.5,50,100,200$, increasing from top to bottom.

Fig. 5. The RG flow lines are illustrated in Fig. 6. We see that in the flat phase at the first stage of the renormalization the bending rigidity strongly decreases, while the disorder strength slightly increases. This behavior indicates the tendency of the membrane to the disorderinduced crumpling. However, below the critical line the disorder strength is insufficient to destroy the membrane, so that $\tilde{\varkappa}$ eventually shows a minimum and then starts to grow, while the disorder gets suppressed. Close to the minimum of $\tilde{\varkappa}$, the RG curve is approximately vertical. This behavior can be interpreted as screening of disorder by softened membrane.

The minimal value of the bending rigidity is given by

$$
\tilde{\varkappa}_{\min } \simeq \varkappa_{\mathrm{cr}}\left\{\begin{array}{l}
1+2 \sqrt{\delta}, \text { for } \delta \rightarrow 0, \\
12 \ln \delta, \text { for } \delta \rightarrow \infty .
\end{array}\right.
$$

A particularly interesting behaviour is predicted slightly above the critical curve $(\delta<0$ with $|\delta| \ll 1)$. In this case, the disorder first increases, then reaches a maximum, starts to decrease and, finally, after reaching a deep minimum, increases again and goes to infinity.

The geometry of membrane is determined by the behavior of the stretching factor $\xi$, which can be expressed in terms of $K(f)$ by using Eqs. (73) and (75):

$$
\xi^{2}=\exp \left[-\int_{f}^{f_{0}} d y \frac{(1+2 y)^{2}}{y(1+3 y) K(y)}\right] .
$$

The value of $\xi$ in the limit $L \rightarrow \infty$ is found from this equation by putting $f=0: \xi_{z=\infty}=\xi_{f=0}$.

Having in mind applications to graphene, let us now discuss in more detail the scale dependence of the bending rigidity and disorder in the flat phase $(\delta>0)$. Similar to the clean case, the behavior of membrane is essentially different deep in the flat phase (when $\delta$ is large) and in the near-critical regime (when $\delta$ is small). The regions corresponding to different regimes are shown schematically in Fig. 7 on the $\left(\delta, f_{0}\right)$ plane. Regions (I), (II), and 


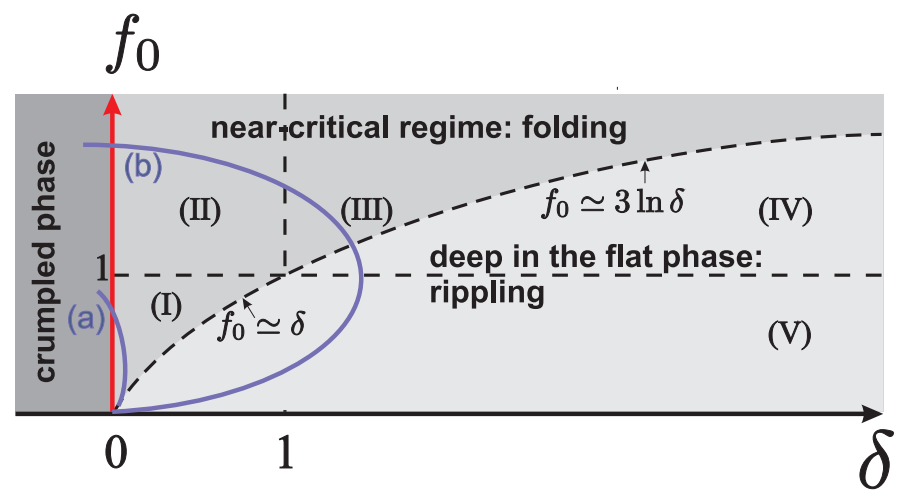

FIG. 7: Phase diagram in the $\left(\delta, f_{0}\right)$ plane. Critical curve (shown in red) separates flat and crumpled phases. Clean case corresponds to horizontal axis $\left(f_{0}=0\right)$. Regions (I), (II), (III) correspond to a near-critical regime within the flat phase. In this part of the phase space, the membrane shows critical (fractal) folding at intermediate scales before flattening at larger scales. Regions (IV) and (V) correspond to a rippled membrane deep in the flat phase. Blue curves correspond to fixed values of bare bending rigidity $\varkappa_{0}$ : (a) $\left(\varkappa_{0}-\varkappa_{\mathrm{cr}}\right) / \varkappa_{\mathrm{cr}} \ll$ $1 ;(\mathrm{b})\left(\varkappa_{0}-\varkappa_{\mathrm{cr}}\right) / \varkappa_{\mathrm{cr}} \gg 1$. Bare disorder increases along these curves from the bottom to the top.

(II) are sufficiently close to critical line, so that the membrane undergoes strong folding in course of the renormalization. Therefore, we term these regions near-critical. In contrast, for membranes with a starting point within regions (IV) and (V), the stretching parameter $\xi$ does not change essentially in course of renormalization (i.e., $\xi$ remains close to unity in the limit $L \rightarrow \infty)$. Such membranes do not fold and only show small dynamical wrinkling and static rippling.

We consider separately the cases of small and large deviations from the critical transition line.

\section{A. Close to critical line [regions (I), (II), and (III) in Fig. 7 .}

When the system is in the flat phase but not far from the transition (i.e, $\delta>0$ but $\delta \ll 1$, regions (I) and (II) in Fig. (7), the dependence $\tilde{\varkappa}(f)$ has a minimum at $f \simeq \sqrt{\delta}$. The minimal value is given by $\left(\tilde{\varkappa} / \varkappa_{\mathrm{cr}}\right)_{\min } \simeq 1+2 \sqrt{\delta}$ and therefore is very close to the critical value for the clean membrane. The disorder strength shows a maximum at a much larger values of $f$, namely, $f \simeq 2$. The maximal value of disorder is given by $\tilde{b}^{\max } d_{c}^{2} / 2 \pi \simeq 1.9$.

For strong disorder, when the starting values of $f$ is large, $f_{0} \gg 1$ [region (II)], the overall RG evolution is particularly rich. At the initial stage of renormalization (i.e., as long as $f$ remains large), we find form Eq. (75) that $f$ changes linearly with $z$ :

$$
f(z)=f_{0}-\frac{3 z}{4}, \quad \text { for } f \gg 1 .
$$

As follows from Eq. (106), at such spatial scales the dis- order slowly increases,

$$
\frac{\tilde{b}(z) d_{c}^{2}}{2 \pi} \simeq 1+\frac{4}{f(z)} .
$$

Here we have taken into account that $\delta \ll 1$, replaced $K$ with $K_{\text {cr }}$ in the denominator of Eq. (106), and used the large- $f$ asymptotic of the function $K_{\text {cr }}$ [see Eq. (98)] ]. The bending rigidity decreases linearly with $z$ :

$$
\frac{\tilde{\varkappa}}{\varkappa_{\mathrm{cr}}} \approx 4 f(z)
$$

The scaling of disorder changes when $f$ becomes of the order of unity. This happens for

$$
z_{r} \approx \frac{4 f_{0}}{3}+O\left[\ln f_{0}\right]
$$

As seen from Eq. (70), for large scales $f$ decays exponentially with $z$, i.e., $f(z) \sim \exp (-z)$, so that the disorder starts to decrease as

$$
\frac{\tilde{b}(z) d_{c}^{2}}{2 \pi} \approx \frac{4 f(z)}{K[f(z)]} .
$$

Although the disorder strength starts to fall, the bending rigidity is still strongly affected by disorder and continues to decrease up to a value $\tilde{\varkappa}_{\text {min }}$ where $f$ becomes quite small (of the order of $\sqrt{\delta}$ ). Only after this the bending rigidity begins to grow.

In the case when the starting value of disorder is weaker, $\sqrt{\delta} \ll f_{0} \ll 1$ [region (I)], it does not show a maximum and monotonously decreases in course of the renormalization. However, as was mentioned in the previous paragraph, the disorder strongly affects $\tilde{\varkappa}$ as long as $f>\sqrt{\delta}$.

The disorder becomes irrelevant for the evolution of bending rigidity for $f_{0} \ll \sqrt{\delta}$. In this case, $f \approx$ $f_{0} \exp (-z)$ and, using Eqs. (96), (98), (99), (105), and (106), we arrive at the following scaling dependencies

$$
\begin{aligned}
& \tilde{\varkappa} \approx \varkappa_{\mathrm{cr}}+\left(\varkappa_{0}-\varkappa_{\mathrm{cr}}\right) e^{z}, \\
& \tilde{b} \approx \frac{b_{0} \varkappa_{0} e^{-z}}{\varkappa_{\mathrm{cr}}+\left(\varkappa_{0}-\varkappa_{\mathrm{cr}}\right) e^{z}} .
\end{aligned}
$$

The disorder strength decreases in course of the renormalization, first as $\exp (-z)$ and, at larger scales, when bending rigidity becomes large enough, as $\exp (-2 z)$. A crossover between these two regimes happens at $f \sim \delta$, i.e. on the boundary separating the near-critical and flat phases.

Using Eq. (108), one can easily find that in regions (I) and (II) the membrane folds in the course of the renormalization. In particular, in the region (II), we find that at the early stage of renormalization (when $f \gg 1$ ) the rescaling parameter $\xi$ is given by $\xi^{2} \approx \exp \left[\left(f_{0}-f\right) / 3\right] \approx$ $\exp [-z / 4]=\left(q^{*} L\right)^{-\eta / 4}$. Hence, first folding of membrane occurs at $z \approx 4$. This yields the following estimate for the length scale of folding:

$$
L_{1} \sim \frac{1}{q^{*}} e^{4 / \eta} .
$$


One can also find that $\xi$ saturates at an exponentially small value

$$
\xi_{z=\infty}^{2}=\frac{\delta}{f_{0}} e^{-f_{0} / 3}
$$

for $L \sim L_{2}$, where

$$
L_{2} \sim \frac{1}{q^{*}}\left(\frac{f_{0}}{\delta}\right)^{1 / \eta} e^{4 f_{0} / 3 \eta}
$$

It is worth stressing that $L_{2}$ coincides (up to a prefactor) with a length scale corresponding to maximum of disorder.

In the region (I) disorder does not essentially affect the folding of the membrane, i.e., the dynamical fluctuations dominate. Therefore, $L_{1}$ and $L_{2}$ are given by Eqs. (94) and (95) derived above for the clean case.

Let us now discuss the region (III), where $f_{0} \gg$ $3 \ln \delta \gg 1$. Using the asymptotic of $K(f)$ at large $f$ [see Eqs. (96) and (98)], we find that the functions $\tilde{\varkappa}(f)$ and $\tilde{b}(f)$ have, respectively, minimum and maximum at $f_{m} \approx 3 \ln \delta$. The minimal value of rigidity is much larger than the critical value, $\tilde{\varkappa}_{\text {min }} / \varkappa_{\text {cr }} \approx 12 \ln \delta$, while the maximal value of disorder slightly exceeds the universal value $2 \pi / d_{c}^{2}$; specifically, $\tilde{b} d_{c}^{2} / 2 \pi-1 \sim 1 / f_{m}$.

In view of the assumption $f_{0}>f_{m}$, the maximum in $\tilde{b}$ is reached at

$$
z_{r}=\frac{4}{3}\left(f_{0}-3 \ln \delta\right)
$$

For $z \ll z_{r}$ the behavior of $\tilde{b}$ and $\tilde{\varkappa}$ can be well described by Eqs. (110) and (111) with $f(z)$ given by Eq. (109). For $z \gg z_{r}$, one can neglect $K_{\mathrm{cr}}$ in expression (96), so that Eqs. (105) and (106) become

$$
\begin{aligned}
\frac{\tilde{\varkappa}}{\varkappa_{\mathrm{cr}}} & =\frac{\delta(3 f+1)^{1 / 9}}{f} e^{-f / 3}, \\
\frac{\tilde{b} d_{c}^{2}}{2 \pi} & =\frac{4 f^{2}}{\delta(3 f+1)^{1 / 9}} e^{f / 3} .
\end{aligned}
$$

Since $f_{m} \gg 1$, there exists a large interval of $z$, where these formulas can be used and at the same time $f \gg 1$, so that Eq. (109) is applicable. In this interval we get, with exponential precision,

$$
\tilde{\varkappa} \propto e^{z / 4}, \quad \tilde{b} \propto e^{-z / 4} .
$$

Finally, for very large $z$, when $f$ becomes smaller than unity and decays as $\exp (-z)$, we find from Eqs. (120) and (121):

$$
\tilde{\varkappa} \propto e^{z}, \quad \tilde{b} \propto e^{-2 z} .
$$

The latter regime is well described by Eqs. (114) and (115) with $\varkappa_{0}-\varkappa_{\mathrm{cr}} \gg \varkappa_{\mathrm{cr}}$.

In analogy with the regions (I) and (II), we find that in the region (III) the membrane undergoes a critical folding in a broad range of length scales, $L_{1}<L<L_{2}$. The value of $L_{1}$ is given by the same equation (116) as in the region (II). The stretching factor saturates at the value

$$
\xi_{z=\infty}^{2}=\delta e^{-f_{0} / 3}
$$

when the spatial scale $L$ becomes of the order of

$$
L_{2} \sim \frac{1}{q^{*}} e^{4\left(f_{0}-3 \ln \delta\right) / 3 \eta} .
$$

Again, up to a prefactor this length coincides with the length $L_{r}$ where disorder has a maximum.

\section{B. Away from the critical line [regions (IV) and} (V) in Fig. 7 .

The evolution of bending rigidity and disorder for a membrane with initial couplings within the region (IV) or (V) coincides with the intermediate (or, respectively, final) stage of renormalization of $\tilde{\varkappa}$ and $\tilde{b}$ for the case of bare couplings in the region (III). Specifically, both $\tilde{\varkappa}$ and $\tilde{b}$ change monotonously with increasing spatial scale: $\tilde{\varkappa}$ increases, while $\tilde{b}$ decreases. For the region (IV) there exists a large interval of length scales $L$ with $1<f<$ $3 \ln \delta$, where Eqs. (120), (121), and (122) apply. With further increase of $L$, we enter the region $f<1$, where scaling dependencies change to Eq. (123). For the region (V) the asymptotic formulas (123) are valid from the very beginning of the renormalization.

Let us analyze the evolution of the stretching factor $\xi$ in these two regions. For region (V) the membrane stretching is fully controlled by dynamical fluctuations, while the disorder is irrelevant. Hence, one can use Eqs. (87) and (88) derived for the clean case. One can easily check that within the region $(\mathrm{V}) \varkappa_{0}-\varkappa_{\mathrm{cr}} \gg \varkappa_{\mathrm{cr}}$, so that $\xi$ does not change essentially and, consequently, the membrane does not fold, remaining approximately flat at all scales. The same statement is valid for region (IV) as well. Indeed, in this case, one can use the inequality $1<f<3 \ln \delta$ and replace the function $K(f)$ in Eq. (108) with $3^{1 / 9} \delta f^{-17 / 9} \exp (-f / 3) \gg 1$ [see Eq. (96) ]. Estimating then the integral in Eq. (108), we find that the renormalization of $\xi$ remains small.

This completes the analysis of the RG flow of the bending rigidity and the disorder for membranes with bare couplings in different regions of the flat phase. We have seen that the evolution can be very non-trivial, with several intermediate scaling regimes. It is worth emphasising, however, that the scaling behaviour at longest scales, $L \sim q^{-1} \rightarrow \infty$, is the same for the whole flat phase: the bending rigidity increases, while the disorder gets suppressed according to universal power laws,

$$
\tilde{\varkappa} \propto L^{\eta}, \quad \tilde{b} \propto L^{-2 \eta} \quad \text { for } L \rightarrow \infty,
$$

with $\eta \simeq 2 / d_{c}$ in the large- $d_{c}$ limit. 


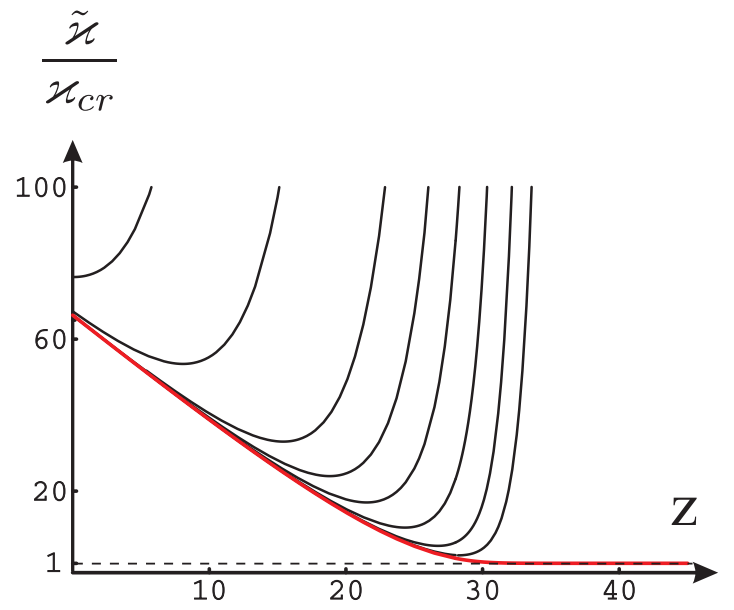

FIG. 8: Scale dependence of the renormalized bending rigidity $\tilde{\varkappa}$ in the flat phase for $\delta=1,5,25,100,300,1000,10000$, 100000 , increasing from bottom to top. Critical curve $(\delta=0)$ is shown in red.

\section{Spatial scale of ripples}

As follows from the above discussion, the scaling dependence of both $\tilde{\varkappa}$ and $\tilde{b}$ in the flat phase are especially non-trivial when the bare disorder is sufficiently strong. In particular, $\tilde{b}$ shows in this case a maximum in the course of renormalization. The spatial scale corresponding to the maximum can be easily found from expression for $z_{r}$ and is given by

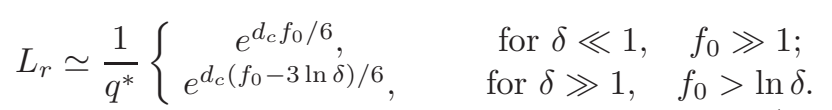

For $L<L_{r}$ the disorder slowly increases with $z$, while for $L>L_{r}$ it decays exponentially with $z$ (i.e., according to a power law with respect to $L$ ). In other words, the disorder is, roughly speaking, "switched off" at $L>L_{r}$. Hence, one can interpret $L_{r}$ as a characteristic scale of random static deformations - ripples. It is worth emphasising at this point that in the case of a nearly critical membrane such "ripples" are multiply folded (fractal) configurations.

When the bare disorder is weaker [regions (IV) and (V) in Fig. 7, the ripples take a more conventional form of relatively small static deformations of a nearly flat surface. Indeed, in this case $\xi \approx 1$, so that the membrane does not fold. For both regions, the suppression of disorder begins already on the Ginzburg length $1 / q^{*}$. The scaling of disorder at the initial stage of renormalization is different for regions (IV) and (V):

$$
b \propto\left(q^{*} L\right)^{-\eta / 4}
$$

for region (IV) and

$$
b \propto\left(q^{*} L\right)^{-2 \eta},
$$

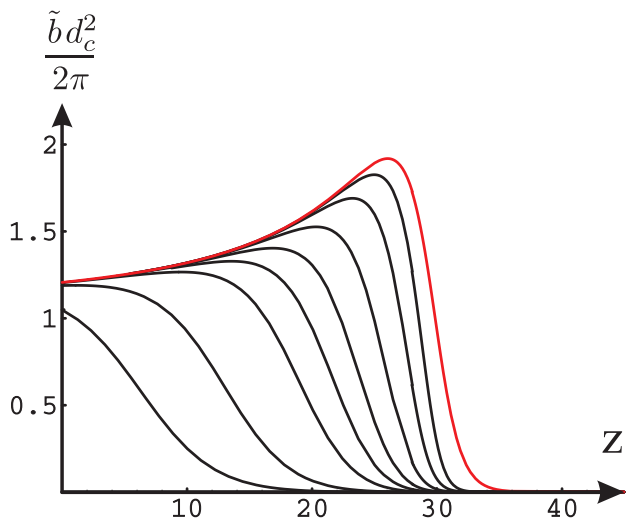

FIG. 9: Scale dependence of the renormalized disorder $\tilde{b}$ in the flat phase for fixed $f_{0}=20$ and for $\delta=1,5,25,100,300$, $1000,10000,100000$, increasing from top to bottom. Critical curve $(\delta=0)$ is shown in red.

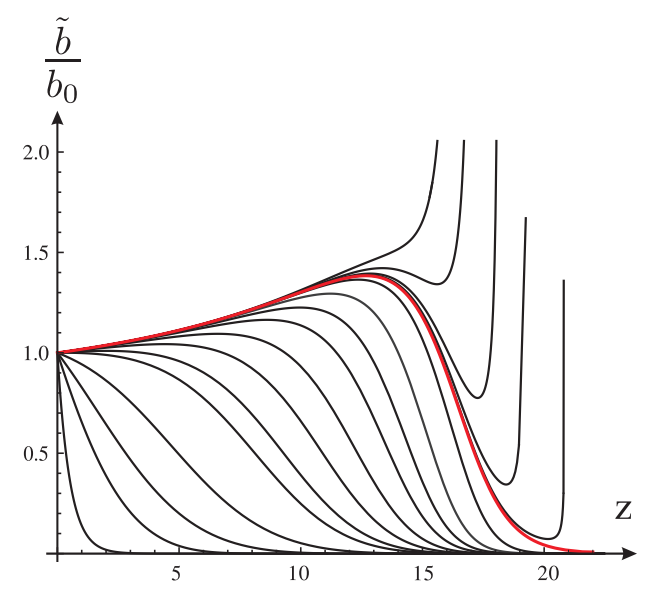

FIG. 10: Scale dependence of the renormalized disorder $\tilde{b}$ (measured in the units of its bare value $b_{0}$ ) for $\varkappa_{0} / \varkappa_{\mathrm{cr}}=$ 30 and for different value of $b_{0} d_{c}^{2} / 2 \pi=0.001,0.5,0.9,1.2$, $1.34,1.36,1.37525,1.382,1.385,1.386,1.38672,1.3867555$, $1.38676,1.38677,1.3868,1.38685$ increasing from bottom to top. Critical curve $\left(\delta=0, b_{0}=1.38675444319\right)$ is shown in red.

for region (V). Hence, the ripple scale can be estimated as

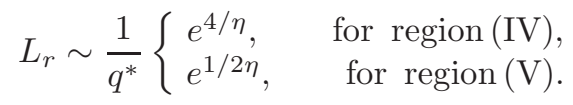

While $\eta$ is small $\left(\eta=2 / d_{c}\right)$ in the limit of high spatial dimensionality of the embedding space, it is a number of order unity, $\eta \simeq 0.7 \div 0.8$, for a physical $2 \mathrm{D}$ membrane (e.g., graphene) in a 3D space. This yields the characteristic scale for ripples of the order of Ginzburg length ${ }^{93}$

The functions $\tilde{\varkappa}(z)$ and $\tilde{b}(z)$ are plotted in Figs. 8 and 9 for $f_{0}=20$ and different values of $\delta$. These figures nicely illustrate the non-monotonous scale dependencies of the coupling constants, with the bending rigidity $\tilde{\varkappa}(z)$ having a minimum and the disorder $\tilde{b}(z)$ showing a maximum, in full agreement with the above analytical results. 


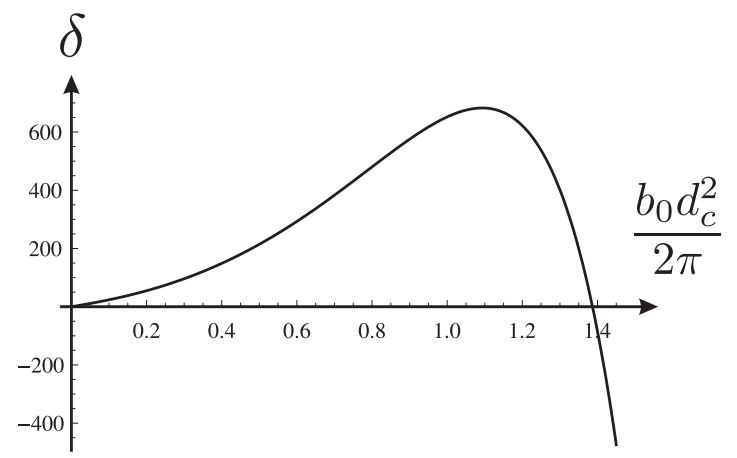

FIG. 11: Dependence of the parameter $\delta$ (which labels RG flow lines) on the bare disorder $b_{0} d_{c}^{2} / 2 \pi$ for fixed bending rigidity, $\varkappa_{0} / \varkappa_{\mathrm{cr}}=30$. The sign change of $\delta$ corresponds to the crumpling transition.

We also plot the dependence of disorder on the spatial scale for a fixed value of $\varkappa_{0}=30 \varkappa_{\mathrm{cr}}$ and various values of the bare disorder $b_{0}$ (see Fig. 10). In this case, starting points of the RG flow lie on a vertical line in Fig. 6. This plot serves as a nice illustration of the dependence of the characteristic scale $L_{r}$ on the bare disorder $b_{0}$. Indeed, it is seen, that for small $b_{0}$ the disorder drops quickly at the Ginzburg scale, in agreement with Eq. (130). With increasing $b_{0}$ the length $L_{r}$ increases. This effect becomes particularly strong when disorder becomes comparable to the critical one, i.e., the parameter $\delta$ approaches zero, as predicted by Eq. (127). For the near-critical curves ( $\delta$ small but still positive) and the critical one $(\delta=0)$, the disorder slowly increases up to a parametrically large scale $L_{r}$ and then drops down. On the other side of the transition $(\delta<0)$, the disorder shows the same behaviour for a while but eventually starts to increase rapidly, which reflects the crumpling. In Fig. 11 we plot the parameter $\delta$ as a function of the bare disorder.

\section{Dynamic and static correlation functions}

To characterize dynamic and static fluctuations in the membrane, we introduce the following correlation functions: $: \underline{6}$

$$
\begin{aligned}
\overline{\left\langle h^{\alpha}(0) h^{\beta}(\mathbf{x})\right\rangle} & =\delta_{\alpha \beta} G^{d+s}(x) \\
\overline{\left\langle h^{\alpha}(0)\right\rangle\left\langle h^{\beta}(\mathbf{x})\right\rangle} & =\delta_{\alpha \beta} G^{s}(x),
\end{aligned}
$$

where angular brackets denote the Gibbs averaging, while the overline stands for the disorder averaging. The function $G^{d+s}$, incorporates both dynamical and static fluctuations, while $G^{s}$ includes static correlations only. These functions depend on the absolute value of the distance $x=|\mathbf{x}|$, so that their Fourier transforms

$$
\begin{aligned}
G_{q}^{d+s} & =\int G^{d+s}(x) e^{-i \mathbf{q} \mathbf{x}} d^{2} \mathbf{x} \\
& =2 \pi \int G^{d+s}(x) J_{0}(q x) x d x, \\
G_{q}^{s} & =\int G^{s}(x) e^{-i \mathbf{q} \mathbf{x}} d^{2} \mathbf{x} \\
& =2 \pi \int G^{s}(x) J_{0}(q x) x d x
\end{aligned}
$$

depend on the absolute value of momentum $q=|\mathbf{q}|$.

The dynamic part of the fluctuations is thus given by the difference of these two functions,

$$
G_{q}^{d}=G_{q}^{d+s}-G_{q}^{s}
$$

(this function was used in the previous sections without index $d$ ). The correlation functions defined above can be straightforwardly calculated on the basis of the above RG analysis. Specifically, we first renormalize the theory from the original ultraviolet scale to the scale $1 / q$. As a result, all non-linear effects get incorporated in the renormalization of $\varkappa$ and $b$. Having renormalized the couplings, we evaluate the correlation functions at the Gaussian level, which yields

$$
\begin{aligned}
G_{q}^{d+s} & =\frac{1}{q^{4}}\left(\frac{T}{\varkappa_{q}}+b_{q}\right), \\
G_{q}^{d} & =\frac{T}{\varkappa_{q} q^{4}}, \\
G_{\mathbf{q}}^{s} & =\frac{b_{q}}{q^{4}},
\end{aligned}
$$

where $\varkappa_{q}$ and $b_{q}$ depend on $q$ according to the RG equations derived above.

At this point, it is worth recalling that the running scale $\Lambda$ for renormalization of $\varkappa$ and $b$ is associated with a wave-vector $\mathbf{q}$ conjugated to the coordinate $\mathbf{x}$ in the reference plane. From the experimental point of view, a more natural coordinate on the membrane surface is given by the vector $\mathbf{r}$ in the embedding space [see Eq. (18)]. As seen from the Fig. 12 a difference between vectors $\mathbf{r}$ and $\mathbf{x}$ becomes stronger when the system approaches criticality.

The physical correlation functions depend on the distance $r=|\mathbf{r}|$ in the embedding space between two points on the membrane surface:

$$
\begin{aligned}
g^{d+s}(r) & =G^{d+s}(r / \xi)=\int g_{Q}^{d+s} J_{0}(Q r) \frac{Q d Q}{2 \pi}, \\
g^{s}(r) & =G^{s}(r / \xi)=\int g_{Q}^{s} J_{0}(Q r) \frac{Q d Q}{2 \pi} \\
g^{d}(r) & =g^{d+s}(r)-g^{s}(r) .
\end{aligned}
$$

Here $\xi=\xi_{x}$ is the running stretching parameter that relates $r$ and $x: r=\xi_{x} x$ [see Eq. (93)]. The correlation 


\section{membrane without fluctuations}

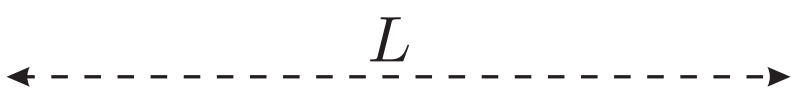

\section{membrane deep in the flat phase}

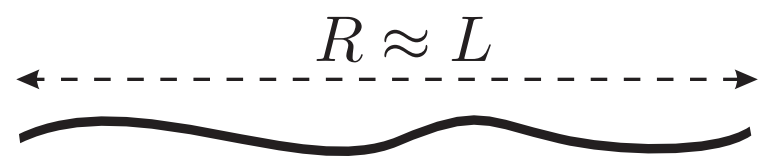

\section{critical membrane}

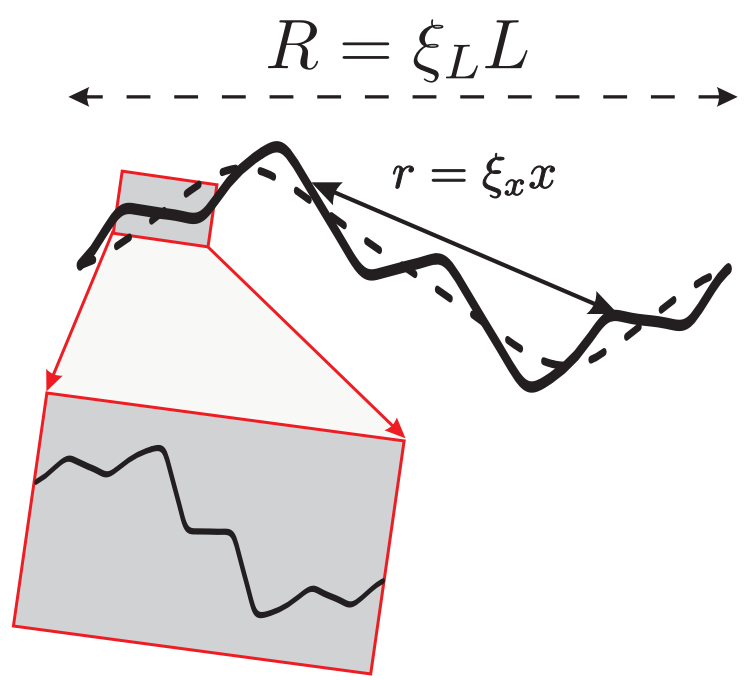

FIG. 12: Top: Membrane with intrinsic size $L$ in the absence of fluctuations; Center: Membrane deeply in the flat phase; Bottom: Membrane at criticality. The linear size, $R$, of membrane with fluctuations is smaller by a factor $\xi_{L}$. The distance, $r$, in the embedding space between two points on membrane surface scales with the distance on the reference plane with a local stretching factor: $r \simeq \xi_{x} x$. Membrane shows self-similarity at different scales, so that the dashed line represents a coarse-grained shape of the membrane at largest scale shown in the picture. The fractal structure of membrane is illustrated in the grey box magnifying a segment of the membrane.

function $g_{Q}$ (measured experimentally) is related to $G_{q}$ as follows:

$$
g_{Q / \xi}=\xi^{2} G_{Q}
$$

with $\xi=\xi_{Q}$. In the flat phase, the rescaling factor $\xi$ remains finite in the infrared limit (i.e. the membrane linear size $R=\xi_{L} L$ in the embedding space is proportional to its intrinsic size $L$ ), and does not essentially affect the scaling. Therefore, the difference between $g$ and $G$ is immaterial. On the other hand, for a critical or near-critical membrane this difference is of crucial importance. In particular, the RG flow is controlled by rescaled couplings $\tilde{\varkappa}$ and $\tilde{b}$ (rather than by $\varkappa$ and $b$.) Further, we find from Eq. (142) that the correlation functions in the embedding space, $g_{Q}^{d+s}, g_{Q}^{d}$, and $g_{Q}^{s}$, are given by Eqs. (136), (137), and (138) with $\varkappa$ and $b$ replaced by $\tilde{\varkappa}$ and $\tilde{b}$, respectively.

In the flat phase, we get the following asymptotic scaling behaviour of the static and dynamic correlation functions at $Q \rightarrow 0$ :

$$
\begin{aligned}
g_{Q}^{d+s} \sim g_{Q}^{d} & \propto \frac{1}{Q^{4-\eta}}, \\
g_{Q}^{s} & \propto \frac{1}{Q^{4-2 \eta}} .
\end{aligned}
$$

It is worth noting that an analogous asymptotic relation between the dynamic and static correlation functions was obtained in Ref. 66 for $D=4-\epsilon$ for the flat-phase fixed point (called P4 point there). Equations (143), (144) imply, in particular, that the characteristic dynamic and static transverse excursions of a membrane (root- meansquare values of the corresponding fluctuations of $\mathbf{h}$ ) scale with its size $R \propto L$ as follows:

$$
\begin{aligned}
& h_{\mathrm{rms}}^{d} \propto R^{1-\eta / 2}, \\
& h_{\mathrm{rms}}^{s} \propto R^{1-\eta} .
\end{aligned}
$$

At the crumpling transition point we have $\tilde{\varkappa}=\varkappa_{\text {cr }}=$ const, $\tilde{b} \propto L^{-\eta}$, and $\xi_{L} \propto L^{-\eta / 2}$. Therefore, the characteristic magnitudes of the transverse excursions of the membrane scale at the transition as

$$
\begin{aligned}
h_{\mathrm{rms}}^{d} & \propto R, \\
h_{\mathrm{rms}}^{s} & \propto R^{(2-2 \eta) /(2-\eta)},
\end{aligned}
$$

and $R \propto L^{1-\eta / 2}$. Since $\varkappa_{\mathrm{cr}}$ is proportional to $d_{c}^{2}$ [see Eq. [86)] the dimensionless coefficient in Eq. (147) turns out to be small, on the order of $1 / \sqrt{d_{c}}$.

\section{E. Ripple intensity and correlations}

It is natural to characterize the intensity and spatial correlations of ripples (i.e., of static transverse deformations) with the static dimensionless correlation function of spatial gradients of out-of-plane displacements. Such function can be expressed in terms of $g^{s}$ as follows

$$
H(r)=\int \frac{d Q}{2 \pi} Q^{3} g_{Q}^{s} J_{0}(Q r)
$$

Using Eq. (142), we rewrite Eq. (149) as

$$
H(r)=\int \frac{d^{2} \mathbf{q}}{(2 \pi)^{2}} \frac{\tilde{b}_{q}}{q^{2}} e^{i \mathbf{q x}} .
$$




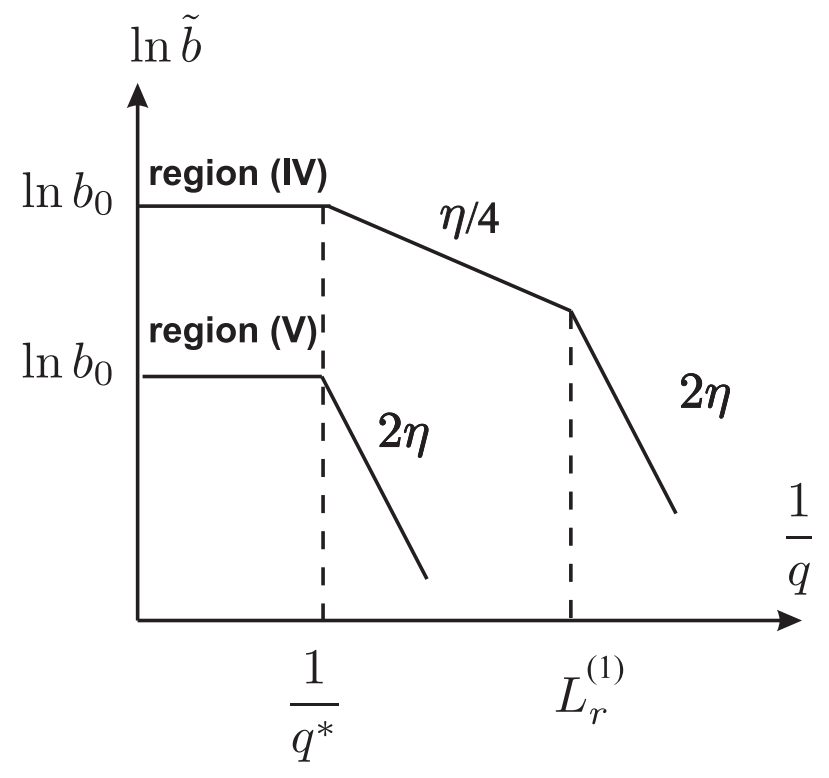

FIG. 13: Schematic dependence of the effective disorder strength $\tilde{b}_{q}$ on the length scale $q^{-1}$ on the log-log scale for regions (IV) and (V). The indices $\eta / 4$ and $2 \eta$ denote the power-law decay exponents in the corresponding regimes.

It is worth noting that function $H(r)$ characterizes fluctuations and correlations of normal vectors to the membrane surface. Such fluctuation of tilt angle of the surface are directly studied in graphene experiments, see below.

Let us now discuss the temperature dependence of the size and height of the ripples deeply in the flat phase, i.e. in regions (IV) and (V) in Fig. 7 . In this situation, the difference between $r$ and $x$ coordinates is not particularly important and can be discarded. Using results of Secs. $\mathrm{VA} \mathrm{VB}$ and $\mathrm{VC}$ we find the behavior of $\tilde{b}_{q}$ (shown schematically in Fig. 13) and thus for $H(r)$ for these two cases.

\section{Strong disorder [region (IV)].}

The calculation of the integral entering Eq. (150) yields

$$
H(r) \sim \frac{b_{0}}{2 \pi}\left\{\begin{array}{cc}
\ln \left(\frac{1}{q^{*} r}\right), & r<1 / q^{*}, \\
\left(\frac{1}{q^{*} r}\right)^{\eta / 4}, & 1 / q^{*}<r<L_{r}^{(1)}, \\
\left(\frac{1}{q^{*} L_{r}^{(1)}}\right)^{\eta / 4}\left(\frac{L_{r}^{(1)}}{r}\right)^{2 \eta}, & L_{r}^{(1)}<r,
\end{array}\right.
$$

In Eq. (151),

$$
L_{r}^{(1)} \sim \frac{1}{q^{*}} e^{4 f_{0} / 3 \eta}=\frac{1}{q^{*}} e^{4 b_{0} \varkappa_{0} / 3 T \eta}
$$

is the spatial scale determined by the condition $f \sim 1$. For $r>L_{r}^{(1)}$, the exponent characterising the spatial de- cay of $H(r)$ changes because of stronger suppression of the disorder by increased bending rigidity. Note that at very small distances, $r<a$ (where $a$ is the ultraviolet cutoff length, which is of the order of the lattice constant), one should replace $r$ with $a$ in the first line of Eq. (151). We thus find that the dimensionless parameter $H(0)$ which represents the averaged squared surface tilt is given by

$$
H(0)=\frac{b_{0}}{2 \pi} \ln \left(\frac{1}{q^{*} a}\right) .
$$

The length $1 / q^{*}$ and the averaged squared tilt $H(0)$ are two natural parameters that characterise the characteristic extension and magnitude of ripples. Importantly, both of them decrease with increasing temperature.

\section{Weak disorder [region (V)].}

In the case of a weak disorder, the only difference is that disorder falls with the exponent $2 \eta$ from the very beginning of renormalization. Hence, similar to the case of strong potential the characteristic ripple size is determined by the Ginzburg scale. For $D=2$ this scale is determined from the condition $3 \mathcal{N} \Pi_{\mathbf{q}} \sim 1$ [see Eq. (33)] ], yielding

$$
q^{*}=q_{\mathcal{N}}^{*} \simeq \sqrt{\frac{3 A_{2} \mu(2 \mu+2 \lambda) T}{(2 \mu+\lambda) \varkappa^{2}}} .
$$

Therefore, we find the ripple size

$$
L_{r} \simeq \frac{2 \pi}{q^{*}} \propto \frac{1}{\sqrt{T}} .
$$

The averaged squared surface tilt is given by Eq. (153) which can be rewritten as

$$
H(0)=\frac{b_{0}}{4 \pi} \ln \left(\frac{T^{*}}{T}\right),
$$

where $T^{*} \simeq \varkappa^{2}(2 \mu+\lambda) /\left[6 A_{2} \mu(\mu+\lambda) a^{2}\right]$. We see that, also in this case, both the size of the ripples $L_{r}$ and the characteristic surface tilt $\sqrt{H(0)}$ decrease with increasing temperature.

\section{Comparison with experiment}

Let us compare our results with available experimental data on suspended graphene. In Ref. 17 the parameters of ripples at room temperature were found to be $L_{r} \simeq 5 \div 10 \mathrm{~nm}$ for the ripple size and $\sqrt{H(0)} \simeq 5^{\circ} \simeq 0.1$ for the characteristic tilt angle. Very similar results at $T=300 \mathrm{~K}$ were obtained in Ref. 81 which found $L_{r} \simeq 10 \mathrm{~nm}$ and $H(0) \simeq 0.01$. The small value of $H(0)$ indicates that the system is in the weak-disorder regime, 
so that the results of Sec. VE2 are expected to be applicable. Indeed, the measured room-temperature ripple size $L_{r}$ agrees well with Eqs. (155) and (154) that yield $L_{r} \simeq 5 \mathrm{~nm}$ at $T=300 \mathrm{~K}$. Comparing (156) with the experimentally measured $H(0) \simeq 0.01$, we get an estimate for the disorder strength, $b_{0} \simeq 0.03$.

The authors of Ref. 81 provided also some information about the temperature dependence of the ripple characteristics. Specifically, they performed measurements also at $T=150 \mathrm{~K}$ and found that, in comparison with the room temperature, the ripple size increased, $L_{r} \simeq 18 \mathrm{~nm}$ whereas the averaged squared surface tilt $H(0)$ remained almost unchanged. These findings are in reasonable agreement with our weak-disorder results (154) and (156) which predict a square-root increase of $L_{r}$ and a slow (logarithmic) increase of $H(0)$ with inverse temperature.

It is worth reminding the reader at this point that static ripples coexist with dynamical fluctuations. In the weak-disorder regime, the relative strength of the two types of fluctuations is controlled by the parameter $f_{0}=b_{0} \kappa_{0} / T$, see Eqs. (137) and (138). According to the above estimate, this parameter is close to unity at room temperature for the samples experimentally studied in Refs. 17 and 81 . However, it has a $1 / T$ temperature dependence which reflects the fact that the dynamical fluctuations become suppressed with lowering temperature. Contrary to this, the typical tilt angle $\sqrt{H(0)}$ characterising static ripples gets enhanced with lowering temperature, Eq. (156). Therefore, measurement of the temperature dependence of $\sqrt{H(0)}$ may be useful for experimentally differentiating between the static and dynamic fluctuations.

\section{SUMMARY AND OUTLOOK}

In this article, we have discussed the rippling and the crumpling transition in graphene with a static quenched disorder. We derived RG equations, Eqs. (68), (69), and (70) for a model of a crystalline membrane with outof-plane (random curvature) disorder. Equations (69) and (70) describe a combined flow of the running dimensionless bending rigidity $\tilde{\varkappa} / T$ and the running disorder strength $\tilde{b} \equiv f T / \tilde{\varkappa}$. They yield, in particular, a critical curve $\tilde{b}(\tilde{\varkappa})$ separating the flat and the crumpled phases, see Figs. 4. 5, and 6. Equation (69) controls the spatial contraction of the membrane due to its deformation. Even deep in the flat phase, random fluctuation of membrane tension caused by the disorder may strongly affect the behavior of the bending rigidity $\tilde{\varkappa}_{q} / T$. Specifically, for a sufficiently strong disorder, the bending rigidity decreases at the first stage of the renormalization, reaches minimum, and only then starts to grow (Fig. 8) . Furthermore, we have found that disorder $\tilde{b}_{q}$ also changes non-monotonously in the flat phase if the bare disorder $b_{0}$ is sufficiently strong. Specifically, $\tilde{b}_{q}$ first increases slowly (logarithmically) with $L$, then reaches a maximum at a certain scale $L_{r}$, and finally decreases according to a power law at larger scales, see Figs. 9 and 10.

The random static out-of-plane fluctuations of the graphene membranes can be identified with experimentally observed ripples, with the length scale $L_{r}$ playing the role of the characteristic ripple size. The found values and temperature dependencies of the ripples parameters - the size $L_{r}$ and the typical surface tilt angle $\sqrt{H(0)}$ - are in a good agreement with experimental observations of Refs. 17, 81 if a disorder strength $b_{0} \simeq 0.03$ is assumed.

We have also briefly discussed an in-plane disorder and showed that it is irrelevant in the RG sense (if one excludes a long-range disorder whose correlation function is highly singular at small momenta) and thus does not affect our main conclusions. The effect of the in-plane disorder at atomic scales may, however, be important for determining the bare value of the out-of-plane disorder.

Before closing the paper, we discuss some of possible directions of future research.

1. In our work we considered a free-standing membrane without tension. On the other hand, the tension may become essential under certain experimental conditions. In a clean case, such a membrane might demonstrate a buckling transition. $\underline{56}$ It remains to be explored what will be the effect of disorder in this situation.

2. We have assumed that the disorder is of shortrange character. On the other hand, a finite density of topological defects (like dislocations or disclinations) may yield long-range-correlated disorder. An earlier work ${ }^{67}$ predicts a variety of possible phases in a membrane with long-range disorder. A study of crumpling transition and of rippling in a membrane with physically relevant long-range disorder remains an interesting prospect for future.

3. Our analysis did not include terms preventing selfcrossing of membrane which are known to become important in the crumpled phase. It remains to be investigated whether such terms may affect the physics in the near-critical regime.

4. There is a certain analogy between the physics of a membrane and that of Anderson metal-insulator transition in disordered (and possibly interacting) systems ${ }^{94,95}$. In particular, the field theory of a disordered membrane developed above bears similarity with the $\sigma$-model description of the Anderson localization. Within this analogy, the flat phase corresponds to a metal, the crumpled phase to an insulator, and the dimensionless bending rigidity $\tilde{\varkappa} / T$ to the dimensionless conductance. Remarkably, both problems manage to evade the MerminWagner theorem, showing a transition also in $D=$ 2. (In the case of Anderson transition, this requires 
either spin-orbit coupling or electron-electron interaction.) Static fluctuations of local deformations (ripples) in a disordered membrane can be viewed a counterpart of mesoscopic fluctuations of wave functions (or local density of states) in the Anderson-localization problem. An interesting and important question is whether this analogy can be pushed further and, in particular, whether the ripple statistics at the crumpling transition is characterised by multifractality that is a hallmark of the Anderson-transition critical point.

5. On the experimental side, a more systematic study of rippling and crumpling in free-standing graphene would be highly desirable. In particular, measurements of the temperature dependence of ripple parameters in a broader temperature range would be of great interest. Furthermore, experiments on various kinds of membranes (including emerging 2D materials) are expected to be instrumental for exploring the whole phase diagram of the problem.

\section{ACKNOWLEDGEMENTS}

We thank L. Radzihovsky for useful discussions. The research was funded by the Russian Science Foundation under the grant No. 14-42-00044.

\section{Appendix A: Applicability of the quasiclassical approximation}

In this Appendix, we provide a justification for the quasiclassical approximation used in this paper and determine the regime of its validity.

The quasiclassical approximation is valid for not too low temperatures (see also Refs 73,74,76 for discussion). More specifically, it is well justified provided that temperature is large compared to frequencies of both out-ofplane and in-plane phonons: $T>\hbar \omega_{\mathbf{q}}, T>\hbar s q$, where $\omega_{\mathbf{q}}$ is given by Eq. (2), and $s=\sqrt{(2 \mu+\lambda) / T}$ is the velocity of the longitudinal in-plane phonons (Here we take account that velocity of transverse in-plane phonons is smaller for graphene parameters.) The characteristic momentum $q$ of the discussed problem is the Ginzburg scale $q_{*}$ which itself depends on temperature. According to Eq. (154), the condition $T>\hbar \omega_{q^{*}}$ can be rewritten as

$$
\rho \varkappa^{3}>36 A_{2}^{2} \hbar^{2} \frac{\mu^{2}(\mu+\lambda)^{2}}{(2 \mu+\lambda)^{2}} .
$$

Note that this inequality does not contain temperature. Substituting graphene parameters $\left(\rho \simeq 7.6 \times 10^{-7} \mathrm{~kg} / \mathrm{m}^{2}\right.$, $\lambda \simeq 3 \mathrm{eV} / \AA^{2}, \mu \simeq 9 \mathrm{eV} / \AA^{2}$, and $\left.\varkappa \simeq 1 \mathrm{eV}\right)$, we find that 1.h.s. of this inequality exceeds the r.h.s. by a factor of the order of $10^{3}$, so that this requirement is perfectly met. This result is not surprising, because the density of graphene $\rho$, entering this estimate is proportional to the atomic mass $M$ and therefore is large compared to typical electronic mass scales. In other words, the l.h.s. of Eq. A1 should be larger than its r.h.s. by a factor of the order of $M / m \sim 2 \cdot 10^{4}$ (where $m$ is the electron mass). Hence, for the problem discussed here, the flexural phonons can be treated semiclassically for any temperature and with a very good precision. The corresponding criterium for longitudinal phonons reads

$$
T>T_{\text {in }}=6 A_{2} \frac{\hbar^{2} \mu(\mu+\lambda)}{\rho \varkappa^{2}} .
$$

The r.h.s. of this inequality can be estimated as $(m / M) E_{a}$, where $E_{a}$ is a characteristic atomic energy scale. Taking $E_{a}=10 \mathrm{eV}$, we get a rough estimate $T_{\mathrm{in}} \simeq 5 \mathrm{~K}$. Using known results for the mechanical parameters of graphene yields a somewhat larger value, $T_{\mathrm{in}} \simeq 80 \mathrm{~K}$, which still leaves enough room for the validity of the semiclassical theory. Furthermore, our theory remains applicable also at lower temperatures, $T<T_{\text {in }}$, where it describes the physics on sufficiently large spatial scales, $q \lesssim T / \hbar s$.

\section{Appendix B: Screening of $h^{4}$ interaction}

In this Appendix, we present technical details of calculation of the screening. As a starting point we use equation for free energy derived in Ref. 65

$$
\begin{aligned}
\frac{F}{T} & =\frac{\varkappa}{2 T} \int(d k) k^{4}\left|\mathbf{h}_{\mathbf{k}}\right|^{2}+\frac{1}{4 d_{c}} \int\left(d k_{1} d k_{2} d k_{3}\right) R_{\alpha \beta \gamma \delta}(\mathbf{q}) \\
& \times k_{1 \alpha} k_{2 \beta} k_{3 \gamma} k_{4 \delta}\left(\mathbf{h}_{\mathbf{k}_{1}} \mathbf{h}_{\mathbf{k}_{2}}\right)\left(\mathbf{h}_{\mathbf{k}_{3}} \mathbf{h}_{\mathbf{k}_{4}}\right),
\end{aligned}
$$

where $\mathbf{q}=\mathbf{k}_{1}+\mathbf{k}_{2}$ and $\mathbf{k}_{1}+\mathbf{k}_{2}+\mathbf{k}_{3}+\mathbf{k}_{4}=0$, and the interaction kernel reads

$$
\begin{aligned}
R_{\alpha \beta \gamma \delta}(\mathbf{q}) & =\frac{\mathcal{N}}{D-1} P_{\alpha \beta} P_{\gamma \delta} \\
& +\mathcal{M}\left(\frac{P_{\alpha \gamma} P_{\beta \delta}+P_{\alpha \delta} P_{\beta \gamma}}{2}-\frac{P_{\alpha \beta} P_{\gamma \delta}}{D-1}\right)
\end{aligned}
$$

Choosing $\mathbf{k}_{1}=\mathbf{k}+\mathbf{q}, \mathbf{k}_{2}=-\mathbf{k}, \mathbf{k}_{3}=-\mathbf{k}^{\prime}-\mathbf{q}, \mathbf{k}_{4}=\mathbf{k}^{\prime}$, we find, after some algebra,

$$
R_{\alpha \beta \gamma \delta}(\mathbf{q}) k_{1 \alpha} k_{2 \beta} k_{3 \gamma} k_{4 \delta}=R_{\mathbf{q}}\left(\mathbf{k}, \mathbf{k}^{\prime}\right),
$$

where $R_{\mathbf{q}}\left(\mathbf{k}, \mathbf{k}^{\prime}\right)$ is given by Eq. (27) of the main text. Screened interaction $\tilde{R}_{\alpha \beta \gamma \delta}$ obeys ${ }^{65}$

$$
\tilde{R}_{\alpha \beta \gamma \delta}=R_{\alpha \beta \gamma \delta}-R_{\alpha \beta \gamma^{\prime} \delta^{\prime}} \Pi_{\gamma^{\prime} \delta^{\prime} \alpha^{\prime} \beta^{\prime}} \tilde{R}_{\alpha^{\prime} \beta^{\prime} \gamma \delta},
$$

with the tensor polarization operator $\Pi_{\gamma \delta \alpha \beta}$ given by

$$
\Pi_{\gamma \delta \alpha \beta}=\int(d k) k_{\alpha} k_{\beta} k_{\gamma} k_{\delta} G_{\mathbf{k}}^{0} G_{\mathbf{q}-\mathbf{k}}^{0} .
$$

Because of the rotation symmetry one can write

$$
\begin{aligned}
& \Pi_{\gamma \delta \alpha \beta}=\left(\delta_{\alpha \beta} \delta_{\gamma \delta}+\delta_{\alpha \gamma} \delta_{\beta \delta}+\delta_{\alpha \delta} \delta_{\beta \gamma}\right) \Pi_{\mathbf{q}} \\
& +\left(\delta_{\alpha \beta} q_{\gamma} q_{\delta}+\cdots\right) \Pi_{\mathbf{q}}^{(1)}+q_{\alpha} q_{\beta} q_{\gamma} q_{\delta} \Pi_{\mathbf{q}}^{(2)}
\end{aligned}
$$


where functions $\Pi_{\mathbf{q}}, \Pi_{\mathbf{q}}^{(1)}$, and $\Pi_{\mathbf{q}}^{(2)}$ depend on $|\mathbf{q}|$ only, and $(+\cdots)$ stands for sum over permutations of $\alpha, \beta, \gamma$, and $\delta$. Due to the projection operators $\hat{P}$ entering in the unscreened coupling, Eq. (B2), functions $\Pi_{\mathbf{q}}^{(1)}$ and $\Pi_{\mathbf{q}}^{(2)}$ drop out from Eq. (B4). The function $\Pi_{\mathbf{q}}$ can be easily obtained by multiplication of Eq. (B5) by $P_{\alpha \beta} P_{\gamma \delta}$ and summation over repeated indices. Taking into account that trace of the matrix $P_{\alpha \beta}$ equals to $D-1$, we arrive at Eq. (37) of the main text. Further, substituting Eq. (B5) into Eq. (B4), we find that the screened interaction $\tilde{R}_{\alpha \beta \gamma \delta}$ can be written in the same form as Eq. (B2) but with $\mathcal{N}$ and $\mathcal{M}$ replaced by their screened values $\mathcal{N}_{\mathbf{q}}$ and $\mathcal{M}_{\mathbf{q}}$. These values are given by Eqs. (33) and (34) of the main text, respectively.

\section{Appendix C: Regularization integrals}

In order to shed light on a connection of our approach to the SCSA, we employ the regularization integrals $A(D, \eta)$ and $B(D, \eta)$ introduced in Ref. 65:

$$
\begin{aligned}
A(D, \eta) & =\frac{1}{D^{2}-1} \int(d x) \frac{x_{\perp}^{4}}{x^{4-\eta}|\mathbf{x}-\mathbf{n}|^{4-\eta}} \quad(\mathrm{C} 1) \\
& =\frac{\Gamma\left(\frac{D+\eta}{2}\right) \Gamma\left(\frac{4-2 \eta-D}{2}\right)}{2^{2 D+\eta+1} \pi^{(D-1) / 2} \Gamma^{2}\left(\frac{4-\eta}{2}\right) \Gamma\left(\frac{1+D+\eta}{2}\right)}, \\
B(D, \eta) & =\int(d x) \frac{x_{\perp}^{4}}{x^{D+2 \eta|\mathbf{x}-\mathbf{n}|^{4-\eta}} \quad(\mathrm{C} 2)} \\
& =\frac{\left(D^{2}-1\right) \Gamma\left(\frac{D+\eta}{2}\right) \Gamma\left(\frac{\eta}{2}\right) \Gamma(2-\eta)}{2^{2+D} \pi^{D / 2} \Gamma\left(\frac{4-\eta}{2}\right) \Gamma\left(\frac{D+2 \eta}{2}\right) \Gamma\left(\frac{4+D-\eta}{2}\right)} .
\end{aligned}
$$

${ }^{1}$ K.S. Novoselov, A.K. Geim, S.V. Morozov, D. Jiang, Y. Zhang, S.V. Dubonos, I.V. Grigorieva and A.A. Firsov, Science 306, 666 (2004).

2 K.S. Novoselov, A.K. Geim, S.V. Morozov, D. Jiang, M.I. Katsnelson, I.V. Grigorieva, S.V. Dubonos and A.A. Firsov, Nature 438, 197 (2005).

3 Y. Zhang, Y.-W. Tan, H.L. Stormer and P. Kim, Nature 438, 201 (2005).

4 A.K. Geim and K.S. Novoselov, Nature Materials 6, 183 (2007).

5 A.H. Castro Neto, F. Guinea, N.M.R. Peres, K.S. Novoselov, and A.K. Geim, Rev. Mod. Phys. 81, 109 (2009).

6 S. Das Sarma, S. Adam, E. H. Hwang, and E. Rossi, Rev. Mod. Phys. 83, 407 (2011)

7 V.N. Kotov, B. Uchoa, V.M. Pereira, F. Guinea, and A. H. Castro Neto, Rev. Mod. Phys. 84, 1067 (2012)

8 M.I. Katsnelson, Graphene: Carbon in Two Dimensions Hardcover, Cambridge University Press (2012)

9 E. L. Wolf, Graphene: A New Paradigm in Condensed Matter and Device Physics, Oxford University Press (2014)

10 L.E.F. Foa Torres, S. Roche, J.-C. Charlier, Introduction to Graphene-Based Nanomaterials From Electronic Struc-
Here $\mathbf{n}$ is an arbitrary unit-length vector and $\mathbf{x}_{\perp}=\mathbf{x}-$ $\mathbf{n}(\mathbf{x n})$. These integrals naturally arise when one uses for calculation of the polarization loop the Green function, Eq. (42), with the self-energy found self-consistently by replacement of the bare Green function $G_{\mathbf{k}}^{0}$ in Eq. (43) with $G_{\mathbf{k}}$. Evaluation of both integrals can be performed in the following way. First, one uses the identity

$$
\begin{aligned}
& \frac{1}{x^{\theta}|\mathbf{x}-\mathbf{n}|^{4-\eta}}=\frac{1}{\Gamma\left(\frac{\theta}{2}\right) \Gamma\left(\frac{4-\eta}{2}\right)} \\
& \times \int_{0}^{\infty} d t_{1} \int_{0}^{\infty} d t_{2} t_{1}^{\theta / 2-1} t_{2}^{1-\eta / 2} e^{-t_{1} x^{2}-t_{2}(\mathbf{x}-\mathbf{n})^{2}},
\end{aligned}
$$

where $\theta=4-\eta$ for the integral $A$ and $\theta=D+2 \eta$ for the integral $B$. The integral over $d x=d \mathbf{x}^{D} /(2 \pi)^{D}$ becomes then a Gaussian one and is easily calculated. The remaining integral can be done by using the following change of variables: $t_{1}=\tau e^{z} \cosh z$ and $t_{2}=\tau e^{-z} \cosh z$. After lengthy but straightforward calculations one arrives at Eqs. (C1) and (C2). ture to Quantum Transport, Cambridge University Press (2014)

11 K.S. Novoselov, Z. Jiang, Y. Zhang, S.V. Morozov, H.L. Stormer, U. Zeitler, J.C. Maan, G.S. Boebinger, P. Kim, and A.K. Geim, Science 315, 1379 (2007).

12 K.I. Bolotin, K.J. Sikes, Z. Jiang, M. Klima, G. Fudenberg, J. Hone, P. Kim, and H.L. Stormer, Solid State Commun. 146, 351 (2008).

13 X. Du, I. Skachko, A. Barker, and E.Y. Andrey, Nature Nanotechnology 3, 491 (2008).

14 K.I. Bolotin, K.J. Sikes, J. Hone, H.L. Stormer, and P. Kim, Phys. Rev. Lett. 101, 096802 (2008).

15 X. Du, I. Skachko, F. Duerr, A. Luican, and E.Y. Andrei, Nature 462, 192 (2009).

16 K.I. Bolotin, F. Ghahari, M.D. Shulman, H.L. Stormer, and P. Kim, Nature 462, 196 (2009).

17 J.C. Meyer, A.K. Geim, M.I. Katsnelson, K.S. Novoselov, T.J. Booth and S. Roth, Nature 446, 60 (2007).

18 J. Scott Bunch, A.M. van der Zande, S.S. Verbridge, I.W. Frank, D.M. Tanenbaum, J.M. Parpia, H.G. Craighead, and P.L. McEuen, Science 315, 490 (2007).

19 F. Miao, S. Wijeratne, Y. Zhang, U.C. Coskun, W. Bao, 
and C.N. Lau, Science 317, 1530 (2007).

20 R. Danneau, F. Wu, M.F. Craciun, S. Russo, M.Y. Tomi, J. Salmilehto, A.F. Morpurgo, and P.J. Hakonen, Phys. Rev. Lett. 100, 196802 (2008).

21 C. Gomez-Navarro, M. Burghard, and K. Kern, Nano Lett. 8, 2045 (2008).

22 D.C. Elias, R.V. Gorbachev, A.S. Mayorov, S.V. Morozov, A.A. Zhukov, P. Blake, L.A. Ponomarenko, I.V. Grigorieva, K.S. Novoselov, F. Guinea, and A.K. Geim, Nature Physics, 7, 701 (2011).

23 C.N. Lau, W. Bao, and J. Velasco, Materials Today 15, 238 (2012).

${ }^{24}$ D. Nelson, T. Piran, and S. Weinberg (Eds.) Statistical Mechanics of Membranes and Surfaces (World Scientific, Singapore, 1989).

25 L.M. Woods and G.D. Mahan, Phys. Rev. B 61, 10651 (2000).

${ }^{26}$ H. Suzuura and T. Ando, Phys. Rev. B 65, 235412 (2002).

27 E.H. Hwang and S. Das Sarma, Phys. Rev. B 75, 205418 (2007)

28 J.L. Manes, Phys. Rev. B 76, 045430 (2007).

29 A.H. Castro Neto and E.A. Kim, Euro. Phys. Lett. 84, 57007 (2008).

30 A. Fasolino, J.H. Los, and M.I. Katsnelson, Nature Materials 6,858 (2007).

31 D.M. Basko and I.L. Aleiner, Phys. Rev. B 77, 041409(R) (2008).

32 D.M. Basko, Phys. Rev. B 78, 125418 (2008).

33 E. Mariani and F. von Oppen, Phys. Rev. Lett. 100, 076801 (2008).

${ }^{34}$ F. von Oppen, F. Guinea, and E. Mariani, Phys. Rev. B 80, 075420 (2009).

35 E. Mariani and F. von Oppen, Phys. Rev. B 82, 195403 (2010).

36 M.A.H. Vozmediano, M.I. Katsnelson, and F. Guinea, Physics Reports 496 109, (2010).

37 E.V. Castro, H. Ochoa, M.I. Katsnelson, R.V. Gorbachev, D.C. Elias, K.S. Novoselov, A.K. Geim, and F. Guinea, Phys. Rev. Lett. 105, 266601 (2010).

${ }^{38}$ K.V. Zakharchenko, R. Roldán, A. Fasolino, and M.I. Katsnelson, Phys. Rev. B 82, 125435 (2010).

39 R. Roldán, A. Fasolino, K.V. Zakharchenko, and M.I. Katsnelson, Phys. Rev. B 83, 174104 (2011).

40 P. San-Jose, J. González, and F. Guinea, Phys. Rev. Lett. 106, 045502 (2011)

41 H. Ochoa, E.V. Castro, M.I. Katsnelson, and F. Guinea, Phys. Rev. B 83, 235416 (2011).

42 I.V. Gornyi, V.Yu. Kachorovskii, and A.D. Mirlin, Phys. Rev. B 86, 165413 (2012).

43 S. Das Sarma and E. H. Hwang, Phys. Rev. B 87, 035415 (2013).

44 M. V. Medvedyeva and Ya. M. Blanter, Phys. Rev. B 88, 125423 (2013).

${ }^{45}$ K. S. Tikhonov, Wei L.Z. Zhao, and A. M. Finkel'stein, Phys. Rev. Lett. 113, 076601 (2014).

46 A. A. Balandin, S. Ghosh, W. Bao, I. Calizo, D. Teweldebrhan, F. Miao, and C. N. Lau, Nano Lett. 8, 902 (2008); A. A. Balandin, Nature Materials 10, 569 (2011).

47 H. Seol, I. Jo, A. L. Moore, L. Lindsay, Z. H. Aitken, M. T. Pettes, X. Li, Z. Yao, R. Huang, D. Broido, N. Mingo, R. S. Ruoff, and L. Shi, Science 328, 213 (2010).

48 Z. Wang, R. Xie, C. T. Bui, D. Liu, X. Ni, B. Li, and J. T. L. Thong, Nano Lett. 11, 113 (2011).

49 K.H. Michel, S. Costamagna, and F.M. Peeters, Phys. Rev.
B 91, 134302 (2015).

50 N. D. Mermin and H. Wagner, Phys. Rev. Lett. 17, 1133 (1966).

51 L.D. Landau and E.M. Lifshitz, Statistical Physics, Part I (Pergamon Press, Oxford, 1980).

52 D.R. Nelson and L. Peliti, J. Phys. (Paris) 48, 1085 (1987).

53 Y.Kantor and D.R. Nelson, Phys. Rev. Lett. 58, 2774 (1987); Phys. Rev. A 36, 4020 (1987);

54 M. Paczuski, M. Kardar, and D.R. Nelson, Phys. Rev. Lett. 60, 2638 (1988)

55 F. David and E. Guitter, Europhys. Lett. 5, 709 (1988).

56 E. Guitter, F. David, S. Leibler, and L.Peliti, Phys. Rev. Lett. 61, 2949 (1988).

57 J.A. Aronovitz and T.C. Lubensky, Phys. Rev. Lett. 60, 2634 (1988).

58 E. Guitter, F. David, S. Leibler, and L. Peliti, J.Phys. France 501787 (1989)

59 J. Aronovitz, L. Golubović, and T.C. Lubensky, J.Phys. France 50609 (1989).

60 M. Paczuski and M. Kardar, Phys. Rev. A 39, 6086 (1989)

${ }^{61}$ L. Radzihovsky and D.R. Nelson, Phys. Rev. A 44, 3525 (1991).

62 D.R. Nelson and L. Radzihovsky, Europhys. Lett. 16, 79 (1991).

${ }^{63}$ G. Gompper and D.M. Kroll, Europhys. Lett. 15, 783 (1991).

64 L. Radzihovsky and P. Le Doussal, J.Phys. I France 2599 (1992).

65 P. Le Doussal and L. Radzihovsky, Phys. Rev. Lett 69, 1209 (1992).

66 D.C. Morse, T.C. Lubensky, and G.S. Grest, Phys. Rev. A 45, R2151 (1992).

67 P. Le Doussal and L. Radzihovsky, Phys. Rev. B 48, 3548 (1993).

68 M.J. Bowick, S.M. Catterall, M. Falcioni, G. Thorleifsson, and K.N. Anagnostopoulos, J. Phys. I France 6, 1321 (1996).

69 J.-P. Kownacki, and D. Mouhanna, Phys. Rev. E 79, 040101(R) (2009).

70 D. Gazit, Phys. Rev. E 80, 041117 (2009).

71 D. Gazit, Phys. Rev. B 80, 161406(R) (2009).

72 F.L. Braghin and N. Hasselmann , Phys. Rev. B 82, 035407 (2010).

73 V.V. Lebedev and E.I. Kats, Phys. Rev. B 85, 045416 (2012).

74 E.I. Kats and V.V. Lebedev, Phys. Rev. B 89, 125433 (2014).

75 B. Amorim, R. Roldán, E. Cappelluti, A. Fasolino, F. Guinea, and M. I. Katsnelson, Phys. Rev. B 89, 224307 (2014).

76 E. I. Kats and V. V. Lebedev, Phys. Rev. E 91, 032415 (2015).

77 A. L. Vázquez de Parga, F. Calleja, B. Borca, M. C. G. Passeggi, Jr., J. J. Hinarejos, F. Guinea, and R. Miranda Phys. Rev. Lett. 100, 056807 (2008).

78 V. W. Brar, Y. Zhang, Y. Yayon, T. Ohta, J. L. McChesney, A. Bostwick, E. Rotenberg, K. Horn, and M. F. Crommie, Appl. Phys. Lett. 91, 122102 (2007).

79 V. Geringer, M. Liebmann, T. Echtermeyer, S. Runte, M. Schmidt, R. Ruckamp, M. C. Lemme, and M. Morgenstern, Phys. Rev. Lett. 102, 076102 (2009).

${ }^{80}$ W. Bao, F. Miao, Z. Chen, H. Zhang, W. Jang, C. Dames, and C. N. Lau, Nature Nanotechnology 4, 562 (2009)

81 D. A. Kirilenko, A. T. Dideykin, and G. Van Tendeloo, 
Phys. Rev. B 84, 235417 (2011)

82 L. Tapasztó, T. Dumitrica, Sung Jin Kim, P. Nemes-Incze, Chanyong Hwang, and L. P. Biró, Nature Physics 8, 739 (2012).

83 D.A. Kirilenko, Technical Physics Letters 39, 325 (2013).

${ }^{84}$ P. Xu, M. Neek-Amal, S. D. Barber, J. K. Schoelz, M.L. Ackerman, P. M. Thibado, A. Sadeghi, and F.M. Peeters, Nature Communications 5, 3720 (2014); M. Neek-Amal, P. Xu, J.K. Schoelz, M.L. Ackerman, S.D. Barber, P.M. Thibado, A. Sadeghi, and F.M. Peeters, ibid 5, 4962 (2014).

85 J. K. Schoelz, P. Xu, V. Meunier, P. Kumar, M. NeekAmal, P. M. Thibado, and F. M. Peeters, Phys. Rev. B 91, 045413 (2015).

${ }^{86}$ C. F. Schmidt, K. Svoboda, N. Lei, I. B. Petsche, L. E. Berman, C. R. Safinya, and G. S. Grest, Science 259, 952 (1993).

87 T. Hwa, E. Kokufuta, T. Tanaka, Phys. Rev. A 44, R2235 (1991); X. Wen, C.W. Garland, T. Hwa, M. Kardar, E. Kokufuta, Y. Li, M. Orkisz, and T. Tanaka, Nature 355 , 426 (1992); C. Cheng and D. Li, Adv. Mater. 25, 13 (2013).

88 S. Costamagna, M. Neek-Amal, J. H. Los, and F. M. Peeters, Phys. Rev. B 86, 041408(R) (2012).

89 R.R. Chianelli, E.B. Prestridge, T.A. Pecoraro, and J.P. Deneufville, Science 203, 1105 (1979); J. Brivio, D.T. L. Alexander, and A. Kis, Nano Lett. 11, 5148 (2011).

90 D. Pacile, J. C. Meyer, C. O. Girit, and A. Zettl, Appl.
Phys. Lett. 92, 133107 (2008).

91 The stability of the SCSA with respect to higher-order contributions was addressed in Ref. 70, where the inclusion of second-order corrections in the parameter $1 / d_{c}=1$ was found to yield $\eta \approx 0.789$ for physical membranes. The SCSA has been applied to graphene in Ref. 38 .

92 As follows from Eqs. (20) and (21), renormalization of $\xi$ starts from the inverse ultraviolet cutoff length, $1 / a$. Here we neglect renormalization of $\xi$ in the interval $q^{*}<q<1 / a$ assuming that $\left(1 / d_{c}\right) \ln \left(1 / q^{*} a\right) \ll 1$.

93 The Ginzburg length as a characteristic scale for thermally excited ripples was also found in Ref. 72. Numerical simulations of graphene flakes performed in Ref. 30 also yielded the spatial scale of ripples close to the Ginzburg length. In Refs. 40 and 71, the size of ripples was related to the coupling of phonons with electrons, yielding a $T$-independent length (ultraviolet atomic scale). Interestingly, taking into account the numerical factors, this scale was estimated ${ }^{71}$ to be close to the thermal Ginzburg scale corresponding to the room temperature. We emphasize that the membrane fluctuations discussed in the above works are dynamical rather than static, see Sec.VD.

94 F. Evers and A.D. Mirlin, Rev. Mod. Phys. 80, 1355 (2008).

95 A.M. Finkelstein, Int. J. Mod. Phys. B 24, 1855 (2010). 